\title{
On the curvature of vortex moduli spaces
}

\author{
Marcel Bökstedt • Nuno M. Romão
}

Received: 28 November 2011 / Accepted: 10 October 2013 / Published online: 14 January 2014

(C) The Author(s) 2014. This article is published with open access at Springerlink.com

\begin{abstract}
We use algebraic topology to investigate local curvature properties of the moduli spaces of gauged vortices on a closed Riemann surface. After computing the homotopy type of the universal cover of the moduli spaces (which are symmetric products of the surface), we prove that, for genus $g>1$, the holomorphic bisectional curvature of the vortex metrics cannot always be nonnegative in the multivortex case, and this property extends to all Kähler metrics on certain symmetric products. Our result rules out an established and natural conjecture on the geometry of the moduli spaces.
\end{abstract}

\section{Introduction}

Gauged vortices $[28,39]$ are of interest as static, stable configurations in various classical gauge field theories with topological solitons. In their simplest realisation, they are defined as solutions $\left(\mathrm{d}_{A}, \phi\right)$ to the vortex equations

$$
\begin{aligned}
\bar{\partial}_{A} \phi & =0, \\
* F_{A}+\mu \circ \phi & =0
\end{aligned}
$$

on a Hermitian line bundle $\mathcal{L} \rightarrow \Sigma$, where $\Sigma$ is a Riemann surface with a Kähler metric represented by the Hodge operator $*$. The "Higgs field" $\phi$ is a smooth section of $\mathcal{L} \rightarrow \Sigma$ and $\bar{\partial}_{A}$ is the $(0,1)$-part of a unitary connection $\mathrm{d}_{A}$ with curvature $F_{A}$. The second equation depends on the choice of a global moment map

$$
\mu: \mathcal{L} \rightarrow \mathfrak{u}(1)^{*} \cong \mathrm{i} \mathbb{R}
$$

M. Bökstedt

Institut for Matematiske Fag,

Aarhus Universitet, Ny Munkegade 118, 8000 Århus C, Denmark

e-mail: marcel@imf.au.dk

N. M. Romão ( $\square)$

Mathematisches Institut, Universität Göttingen, Bunsenstraße 3-5, 37073 Göttingen, Germany

e-mail: nromao@uni-math.gwdg.de 
for the Hamiltonian action of the structure group on the fibres (each equipped with the standard, U(1)-invariant symplectic form), which we fix to be, in a unitary trivialisation,

$$
w \mapsto \mu(w)=\frac{i}{2}\left(\|w\|^{2}-\tau\right),
$$

where $\tau \in \mathbb{R}$. Both vortex equations (1) and (2) are invariant under the group of (unitary) gauge transformations $\left(\mathrm{d}_{A}, \phi\right) \mapsto\left(\mathrm{d}_{A}+u^{-1} \mathrm{~d} u, u^{-1} \phi\right), u \in \operatorname{Aut}_{\Sigma}(\mathcal{L}) \cong C^{\infty}(\Sigma, \mathrm{U}(1))$, and one is usually interested in solutions up to this action. For most of the discussion in this paper, we shall consider $\Sigma$ to be a connected, oriented, closed surface of genus $g \geq 1$, and sometimes we will denote it by $\Sigma_{g}$ for emphasis. Although our focus will be on line bundles, the vortex equations above make sense in much broader contexts, and this has been used to compute and generalise the Gromov-Witten invariants $[3,13]$.

The positive integer $k:=\frac{1}{2 \pi \mathrm{i}} \int_{\Sigma} F_{A}$ expresses the first Chern class $c_{1}(L) \in H^{2}(\Sigma ; \mathbb{Z}) \cong$ $\mathbb{Z}$ in units of the fundamental class, and it can be thought of as a quantised magnetic charge or vortex number. So $k$ is the degree of the effective divisor associated to the holomorphic section $\phi$, whose zeroes (counting multiplicities) correspond to locations of individual vortex cores on the surface. It follows directly from (2) that a metric on $\Sigma$ can only support $k$ vortices provided $\tau \operatorname{Vol}(\Sigma) \geq 4 \pi k$, independently of the choice of Hermitian structure on the line bundle. (We use $\operatorname{Vol}(\Sigma)$ to denote the total area of the surface.) Conversely, Bradlow [11] and García-Prada [19] showed that, if the strict inequality

$$
\tau \operatorname{Vol}(\Sigma)>4 \pi k
$$

is satisfied, one can construct a vortex solution from any effective divisor of degree $k$. Thus the moduli space $\mathcal{M}_{k, g}$ of $k$-vortex solutions on $\Sigma=\Sigma_{g}$ up to gauge transformations is the symmetric product

$$
\mathcal{M}_{k, g} \cong \operatorname{Sym}^{k}\left(\Sigma_{g}\right):=\left(\Sigma_{g}\right)^{k} / \mathfrak{S}_{k} .
$$

(Here $\mathfrak{S}_{k}$ denotes the symmetric group on $k$ letters, acting on the Cartesian product $\left(\Sigma_{g}\right)^{k}$ by permuting the factors.) All these spaces are smooth manifolds, equipped with a natural complex structure induced from the one on $\Sigma_{g}$. We will always assume the parameter $\tau$ to be fixed and to satisfy $\tau>\frac{4 \pi k}{\operatorname{Vol}(\Sigma)}$, whenever we refer to a moduli space of vortices.

In gauge theory, the result (5) already has interesting consequences at the purely topological level (see [15] for an application); but it turns out that the moduli spaces of vortices have a richer structure, as they also support natural Riemannian metrics. On the tangent space to a vortex represented by $\left(\mathrm{d}_{A}, \phi\right)$, one can define this metric pointwise as the restriction of the $L^{2}$-metric to solutions $(\dot{A}, \dot{\phi})$ of the linearised vortex equations about $\left(\mathrm{d}_{A}, \phi\right)$ orthogonal to the orbit of the gauge group. One can show that the natural complex structure is an isometry of this metric, and the two define a Kähler structure [46]. Alternatively, one can understand these Kähler structures in terms of the symplectic (or Kähler) reduction of the $L^{2}$-metric on the affine space of all fields, with respect to the Hamiltonian action of the group of gauge transformations [19].

The geometry of the vortex metrics has been a focus of interest, since it has a whole range of consequences to the physical behaviour of vortices, which is very hard to study otherwise. For instance, the geodesic flow associated to these metrics gives a good approximation to slow scattering in the abelian Higgs model [52], and the corresponding symplectic form is relevant to construct other dynamical systems with potentially interesting applications [14, 35]. Natural extensions of these basic mechanical models might include fermions, or extra potential terms that are covariant with respect to the moduli space metrics, in order to model particular phenomenology. Such extra terms will typically be nonlinear and involve the 
curvature of the Levi-Civita connection associated to the metric on the moduli space [43]. More generally, curvature properties have implications for spectral geometry, which becomes relevant when one goes on to discuss supersymmetric quantum mechanics, or to construct improved approximations to the dynamics in the underlying classical field theories.

Computing the metrics on the moduli spaces is still a very difficult task. Samols [46] was able to write down a local formula in terms of the moduli, using a remarkable localisation argument in the spirit of previous work by Strachan [51]. From a version of Samols' formula for closed surfaces, one has been able to extract some global information-for example, the symplectic volume [36] of the moduli spaces, their total scalar curvature [2], and other integrals [45] which provide information about vortex interactions [16]. All these quantities are determined by the Kähler class; in contrast, qualitative information about the local geometry, such as more detailed curvature properties, has proven more difficult to obtain. In [38], Manton and Speight described two alternative schemes of studying vortex metrics in the regime of large separation among $k \geq 2$ vortex cores on the Euclidean plane $\Sigma=\mathbb{C}$. These yield an asymptotic Kähler metric whose coefficients can be written in terms of Bessel functions, and from it one calculates the corresponding Ricci tensor. This can be seen to be positive semi-definite (at every point), with two-dimensional kernel consisting of the directions associated to rigid translations, and it leads to a scalar curvature Scal which is strictly positive everywhere.

In this paper, we address curvature properties of the moduli spaces that are consistent with the topological consequences of the result (5), and which are in a sense stronger than the sign of the Ricci curvature. The sectional curvature tensor carries all the curvature information about a Riemannian manifold, but in complex geometry it is natural to restrict it to tangent 2-planes that are invariant under the complex structure $J$, and this is what is called the holomorphic sectional curvature. Particularly suitable for considerations relating topology to curvature in Kähler geometry is the holomorphic bisectional curvature introduced by Goldberg and Kobayashi [22], which carries more information than the holomorphic sectional curvature but less information than the sectional curvature. Following the conventions in [42], it is defined for $J$-invariant 2-planes $\sigma, \sigma^{\prime}$ in the tangent space at a point by

$$
B\left(\sigma, \sigma^{\prime}\right):=\frac{R\left(X, J X, X^{\prime}, J X^{\prime}\right)}{\langle X, X\rangle\left\langle X^{\prime}, X^{\prime}\right\rangle},
$$

where $X, X^{\prime}$ are nonzero real vectors contained in $\sigma, \sigma^{\prime}$, respectively, and $\langle\cdot, \cdot\rangle$ denotes the Kähler metric. (Clearly, this definition is independent of the real vectors chosen.) With respect to a basis of normal holomorphic vector fields containing vectors $\xi, \xi^{\prime}$ along $\sigma, \sigma^{\prime},(6)$ can also be described as the component $R_{\xi \bar{\xi} \xi^{\prime} \bar{\xi}^{\prime}}$ of the Riemann tensor in holomorphic coordinates. It is easy to see [22] that positivity (or negativity) of the holomorphic bisectional curvature implies positivity (or negativity) of both the Ricci tensor and the holomorphic sectional curvature, the latter being simply given by $H(\sigma)=B(\sigma, \sigma)$.

We say that a Kähler metric has nonnegative holomorphic bisectional curvature if $B$ is positive or zero at every point, for all choices of $J$-invariant 2-planes. Our main result is the following:

Theorem 1.1 If $g>1$ and $1 \leq k \leq 2 g-2$, no Kähler metric on $\operatorname{Sym}^{k}\left(\Sigma_{g}\right)$ can have nonnegative holomorphic bisectional curvature.

Of course, this result applies to the $L^{2}$-metric of vortices described above and is independent of the choice of metric and complex structure on $\Sigma$, as well as of the Hermitian inner product on $\mathcal{L}$. Theorem 1.1 is somehow unexpected in the light of what is known about 
the curvature behaviour of related moduli spaces with their natural Kähler structures. For example, the moduli spaces of Higgs bundles over a compact Riemann surface carry natural metrics [25]. Fibre-integral type formulas for their curvature tensors have been obtained using deformation theory in terms of representatives of Kodaira-Spencer classes, from which one can deduce that these metrics do have nonnegative holomorphic sectional curvature [8]. Similar positivity results have been established for the curvature of natural metrics on moduli spaces of unitary bundles and holomorphic vector bundles over a compact Riemann surface [7, 9]. All these results build up on earlier work of Itoh on the geometry of spaces of anti-selfdual connections [27]; see also Takhtajan and Zograf [53] and Jost and Peng [29].

An immediate application of Theorem 1.1 is that it rules out a natural conjecture of Biswas and Schumacher stated in Ref. [10] as Corollary 8.2 (without proof); the authors assert that a related metric on the moduli space of stable triples $\left(\mathcal{E}_{1}, \mathcal{E}_{2}, \varphi\right)$ associated to solutions of coupled vortex equations $[12,20]$ should have nonnegative holomorphic bisectional curvature. Here, $\mathcal{E}_{i}(i=1,2)$ are holomorphic vector bundles over a closed Riemann surface $\Sigma$ and $\varphi: \mathcal{E}_{2} \rightarrow \mathcal{E}_{1}$ an $\mathcal{O}_{\Sigma}$-linear holomorphic map. If one takes $\mathcal{E}_{2}$ to be the trivial vector bundle, $\varphi$ can be identified with a section $\phi: \Sigma \rightarrow \mathcal{E}_{1}$ (which determines the morphism $\varphi$ by fibrewise multiplication) satisfying (1), and the coupled vortex equations reduce to the vortex equation (2). Thus gauged vortices are recovered as a special case, and our result above provides counter-examples of this conjecture in the nontrivial multivortex case. In fact, in the case $k=1$, for which $\mathcal{M}_{1}$ (as a complex manifold) is $\Sigma$ itself, and assuming $g \geq 2$, this assertion also follows trivially from the theorem of Gauß-Bonnet, since the Gauß curvature and the holomorphic bisectional curvature on a Riemann surface can easily be seen to coincide. For this reason, our interest will be strictly in the situation where $k>1$.

The strategy of proof of Theorem 1.1 in our paper is the following. If $\operatorname{Sym}^{k}\left(\Sigma_{g}\right)$ had a Kähler metric with nonnegative holomorphic bisectional curvature, so would its universal cover $\operatorname{Sym}^{k}\left(\Sigma_{g}\right)$. Using classification results (specifically, "rigidity" results obtained by Mok in the 1980s, cf. [42]), this would imply that all homology groups of the universal covering space would be finitely generated. But we can compute these groups using algebraic topology, and it turns out that, if $1<k \leq 2 g-2$, the middle homology groups $H_{k}\left(\widehat{\operatorname{Sym}^{k}\left(\Sigma_{g}\right)} ; \mathbb{Z}\right)$ are not finitely generated.

It was pointed out to us that an alternative proof of Theorem 1.1 can be presented, based on results obtained by Fang [17] on the characterisation of compact Kähler manifolds whose holomorphic bisectional curvature satisfies a condition that is weaker than the notion of nonnegativity used in the present paper. This argument explores the fact that the Albanese variety [23] of $\operatorname{Sym}^{k}(\Sigma)$ coincides with the Jacobian variety of $\Sigma$. General properties of Abelian varieties then imply that, if $\operatorname{Sym}^{k}(\Sigma)$ had nonnegative holomorphic bisectional curvature, it would have to be a holomorphic fibration over this Jacobian, as a consequence of Fang's Theorem C in [17]; but this leads to a contradiction for the range of values of $k$ considered in the hypothesis of Theorem 1.1. Since the argument of proof sketched in the preceding paragraph is more constructive, in the sense that it produces new information on the topology of vortex moduli spaces as by-product, we believe that our preference for the strategy of proof using Mok's rigidity results, as given in the present paper, is not unjustified. We are also aware of [6].

Remark 1.2 There is another point of view on the calculations in this paper. Our main strategy to prove Theorem 1.1 is to study the topology of the universal cover of the moduli space of vortices-and this cover has independent interest, since it plays an important role at the level of quantisation. To be more precise: the structure of supersymmetric quantum 
field theories is reflected in the topology and the Hodge theory of the universal covers of their classical moduli spaces. One famous instance of this is provided by the beautiful Sen conjectures [49]. There is a purely mathematical formulation of these conjectures, which amounts to precise statements about $L^{2}$-cohomology of the universal covers of the moduli spaces of centred magnetic monopoles in $\mathbb{R}^{3}$. The dimension of $L^{2}$-cohomology groups gives information about the spectrum of bound states at the lowest energy level, which can therefore be extracted directly from the underlying classical field theory. A variety of methods has been used to prove part of these assertions $[26,47,48,50]$. There is a striking analogy between this body of results and some of the topological underpinnings of our paper. In particular, from Theorem 4.7 below one can establish vanishing results for certain $L^{2}$-Betti numbers and then, using standard techniques [32], compute the nontrivial $L^{2}$-Betti numbers of the moduli spaces we are considering is this paper. In ongoing work, we study the $L^{2}$-cohomology for vortices in the same spirit as the papers $[47,48]$ mentioned above address the Sen conjectures for monopoles.

Let us present the layout of this paper. We start by sketching standard topological results on the symmetric products of a closed oriented surface in Sect. 2. There is a cell decomposition of $\operatorname{Sym}^{k}(\Sigma)$ due to Kallel and Salvatore; in Sect. 3 we recall this construction, and show how to lift it to obtain a cell decomposition of the universal cover, for which we can write down the cellular chain complex. In Sect. 4, we do the algebraic computation that calculates the homology groups of this chain complex. For completeness, we determine the homotopy type of the universal cover in Sect. 5. In Sect. 6 we conclude the proof of Theorem 1.1, and make some final remarks about vortices in Sect. 7.

Remark 1.3 After this work was completed, Indranil Biswas wrote a paper [5] proving that the hypothesis $k \leq 2 g-2$ in our Theorem 1.1 is in fact unnecessary, using arguments in algebraic geometry which apply strictly to the case $k>2 g-2$.

\section{Symmetric products of a closed oriented surface}

For a smooth closed oriented surface $\Sigma$ and a positive integer $k$, the $k$-fold symmetric product

$$
\operatorname{Sym}^{k}(\Sigma):=\Sigma^{k} / \mathfrak{S}_{k}
$$

is a smooth, compact $2 k$-dimensional manifold. If $\Sigma$ is a complex curve (i.e. is given a complex structure), then $\operatorname{Sym}^{k}(\Sigma)$ inherits a complex structure, and it parametrizes degree- $k$ effective divisors on $\Sigma$. This justifies the importance of these spaces in classical algebraic geometry, and also why they appear as moduli spaces of $k$-vortices on $\Sigma$. Our main goal in this paper is to prove a curvature property for Kähler metrics on these manifolds which is implied by the topology of their universal covers. To begin with, we shall recall some standard facts about the topology of the symmetric products themselves, and make some preliminary observations that situate our main topological results within the algebraic-geometric context.

The cohomology ring of $\operatorname{Sym}^{k}(\Sigma)$ was computed by Macdonald [33], and we will quote the result here for completeness. Suppose that $\Sigma \equiv \Sigma_{g}$ has genus $g \geq 1$. Start by fixing a symplectic basis $\left\{x_{1}, \ldots x_{g}, y_{1}, \ldots, y_{g}\right\}$ for $H_{1}\left(\Sigma_{g} ; \mathbb{Z}\right) \cong \mathbb{Z}^{2 g}$, i.e. 1-cycles for which the relations

$$
\sharp\left\langle x_{i}, y_{j}\right\rangle=\delta_{i j}, \quad \sharp\left\langle x_{i}, x_{j}\right\rangle=\sharp\left\langle y_{i}, y_{j}\right\rangle=0
$$

for the intersection pairing are satisfied. (This basis is unique up to a base change in $\mathrm{Sp}_{2 g} \mathbb{Z}$.) The Poincaré-dual basis $\left\{\alpha_{i}\right\}_{i=1}^{2 g}$ of $H^{1}\left(\Sigma_{g} ; \mathbb{Z}\right)$ then generates the integral cohomology ring 
of $\Sigma_{g}$, where we use labels $(\bmod 2 g)$ such that all cup products are determined by the relations

$$
\begin{gathered}
\alpha_{i} \alpha_{j}=0, \quad i \neq j \pm g, \\
\alpha_{i} \alpha_{i+g}=-\alpha_{i+g} \alpha_{i}=\beta, 1 \leq i \leq g,
\end{gathered}
$$

where $\beta \in H^{2}\left(\Sigma_{g} ; \mathbb{Z}\right)$ is the fundamental class (see [18]). Macdonald shows [33] that $H^{*}\left(\operatorname{Sym}^{k}\left(\Sigma_{g}\right) ; \mathbb{Z}\right) \cong H^{*}\left(\left(\Sigma_{g}\right)^{k} ; \mathbb{Z}\right)^{\mathfrak{S}_{k}}$, and this is described as follows. For each $1 \leq \ell \leq k$, let $\pi_{\ell}:\left(\Sigma_{g}\right)^{k} \rightarrow \Sigma_{g}$ denote the projection onto the $\ell$ th factor and consider the classes

$$
\alpha_{i \ell}:=\pi_{\ell}^{*} \alpha_{i}, \quad \beta_{\ell}:=\pi_{\ell}^{*} \beta \quad \in H^{*}\left(\left(\Sigma_{g}\right)^{k} ; \mathbb{Z}\right) \cong H^{*}\left(\Sigma_{g} ; \mathbb{Z}\right)^{\otimes k},
$$

which satisfy the following relations in degree two:

$$
\begin{array}{cl}
\alpha_{i \ell} \alpha_{j \ell}=0 & i \neq j \pm g, \\
\alpha_{i \ell} \alpha_{i+g, \ell}=-\alpha_{i+g, \ell} \alpha_{i \ell}=\beta_{\ell}, & 1 \leq i \leq g, \\
\alpha_{i \ell}, \alpha_{j, \ell^{\prime}}=-\alpha_{j \ell^{\prime}} \alpha_{i \ell}, & \ell \neq \ell^{\prime} .
\end{array}
$$

We sum over the label $\ell$ and obtain the classes

$$
\begin{aligned}
\xi_{i} & :=\sum_{\ell=1}^{k} \alpha_{i \ell}, \quad 1 \leq i \leq 2 g, \\
\eta & :=\sum_{\ell=1}^{k} \beta_{\ell}
\end{aligned}
$$

which descend to $H^{*}\left(\operatorname{Sym}^{k}\left(\Sigma_{g}\right) ; \mathbb{Z}\right)$ since they are $\mathfrak{S}_{k}$-invariant. For convenience, we also define

$$
\sigma_{i}:=\xi_{i} \xi_{i+g}, \quad 1 \leq i \leq g
$$

Then we have $[4,33]$ :

Theorem 2.1 The integral cohomology ring $H^{*}\left(\operatorname{Sym}^{k}(\Sigma) ; \mathbb{Z}\right)$ is generated by the degree-2 class $\eta$ and the degree-1 classes $\xi_{1}, \ldots \xi_{2 g}$ (anticommuting with each other and commuting with $\eta$ ). For each choice of disjoint subsets $I_{1}, I_{2}, J \subset N_{g}:=\{1, \ldots, g\}$, there is an extra relation

$$
\eta^{r} \prod_{i_{1} \in I_{1}} \xi_{i_{1}} \prod_{i_{2} \in I_{2}} \xi_{i_{2}+g} \prod_{j \in J}\left(\eta-\sigma_{j}\right)=0,
$$

for each $r \in \mathbb{N}_{0}$ satisfying

$$
r \geq k-\left|I_{1}\right|-\left|I_{2}\right|-2|J|+1 .
$$

If $k>2(g-1)$, all relations (8) are generated by the one with $J=N_{g}$ and $r=k-2 g+1$. If $k \leq 2(g-1)$, they are generated by those for which $r \in\{0,1\}$ and equality holds in (9).

Of course, the homology of $\operatorname{Sym}^{k}(\Sigma)$ can be read off from this result by Poincaré duality, since these spaces are orientable as complex manifolds. A more refined approach to this calculation was presented by Kallel and Salvatore [31], where a certain homotopy equivalence $\operatorname{Sym}^{k}(\Sigma) \rightarrow \overline{\operatorname{Sym}^{k}(\Sigma)}$ is introduced via a quotienting procedure, together with an explicit cell decomposition of $\overline{\operatorname{Sym}^{k}(\Sigma)}$; then the cell homology of the quotient space can be used to compute the homology of the symmetric products themselves. The Kallel-Salvatore construction will play an important rôle in our discussion, and we shall review it in Sect. 3.

To understand the structure of the universal covers $\widetilde{\operatorname{Sym}^{k}(\Sigma)}$, one might start by fixing a complex structure on $\Sigma$, together with a basepoint $p \in \Sigma$ and a basis of holomorphic 
1-forms. These data define an Abel-Jacobi map [23] for each $k \geq 1$,

$$
\operatorname{Sym}^{k}(\Sigma) \longrightarrow \operatorname{Jac}(\Sigma)=H^{0}\left(\Sigma, K_{\Sigma}\right)^{*} / H_{1}(\Sigma ; \mathbb{Z}),
$$

which can be interpreted as mapping divisors of degree $k$ on $\Sigma$ to equivalence classes of holomorphic line bundles. Holomorphic sections of these bundles (modulo multiplication by scalars) vanish at the $k$ points the divisors specify, counted with multiplicities. If $k>$ $2 g-2=\operatorname{deg} K_{\Sigma}$, then there are no special divisors of degree $k$ (i.e. all divisors correspond to invertible sheaves with vanishing first cohomology group). Riemann-Roch then implies that the corresponding spaces of global sections have dimension $k-g+1$. Thus we can interpret the pre-image of each line bundle via the map (10) as a space of sections modulo scalar multiplication; so the Abel-Jacobi map is a fibre bundle with typical fibre $\mathbb{C P}^{k-g}$ in this situation. The Jacobian variety $\operatorname{Jac}(\Sigma)$ is a real $2 g$-torus, and one can draw the diagram

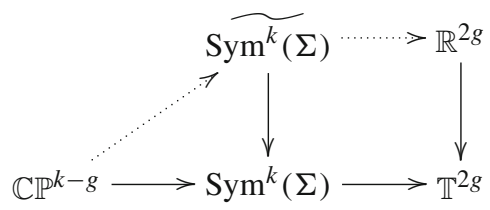

(where the dashed maps exist by the elementary lifting criterion of covering maps [24]). Thus we conclude that for $k>2 g-2$ the universal cover $\widetilde{\operatorname{Sym}^{k}(\Sigma)}$ is itself a $\mathbb{C P}^{k-g}$-bundle over a contractible space, and it has therefore the same homotopy type as the fibres. (Notice that $\operatorname{Sym}^{k} \Sigma_{0} \cong \mathbb{C P}^{k}$, which is simply connected, so this result is trivially true for $g=0$.)

However, when $k \leq 2 g-2$ this analysis no longer applies and the topology is more complicated, as the dimension of the fibres of (10) will jump on special divisors. We shall establish that, in this case, the universal cover $\operatorname{Sym}^{k}(\Sigma)$ turns out to have the same homotopy type as the wedge sum of a complex projective space (whose dimension may be larger than $k-g$ ) and an infinite bouquet of $k$-spheres - cf. Theorem 5.4.

A basic fact that we shall use below is the following result:

Proposition 2.2 For all $k>1, \pi:=\pi_{1}\left(\operatorname{Sym}^{k}\left(\Sigma_{g}\right)\right) \cong H_{1}\left(\Sigma_{g} ; \mathbb{Z}\right) \cong \mathbb{Z}^{2 g}$.

A proof by M. Roth was presented in [4]. This involves looking at the map $\Sigma \rightarrow \operatorname{Sym}^{k}(\Sigma)$ given by $z \mapsto z+(k-1) p$ (for a fixed basepoint $p \in \Sigma$ ) and showing that it induces a surjection of fundamental groups, with Abelian image and kernel $\left[\pi_{1}(\Sigma), \pi_{1}(\Sigma)\right]$.

In the rest of this paper, we will always use $x_{1}, \ldots, x_{g}, y_{1}, \ldots, y_{g}$ to denote generators of the fundamental group $\pi$ of the symmetric product $\operatorname{Sym}^{k}\left(\Sigma_{g}\right)$. These generators can be regarded as images of a standard basis for $H_{1}\left(\Sigma_{g} ; \mathbb{Z}\right)$ as in $(7)$ under the isomorphism in Proposition 2.2.

\section{A cell decomposition of the universal cover}

In [31], Kallel and Salvatore define a quotient map $r: \operatorname{Sym}^{k}(\Sigma) \rightarrow \overline{\operatorname{Sym}^{k}(\Sigma)}$, where $\Sigma=\Sigma_{g}$ is a closed oriented surface of genus $g$. They prove that this quotient map is a homotopy equivalence, and give a cell decomposition of $\overline{\operatorname{Sym}^{k}(\Sigma)}$. They use this cell decomposition to compute the homology of $\overline{\operatorname{Sym}^{k}(\Sigma)}$, and thus the homology of $\operatorname{Sym}^{k}(\Sigma)$.

Our main goal in this section is to explain how this cell decomposition can be lifted to study the universal covers $\widetilde{\operatorname{Sym}^{k}(\Sigma)}$, and to describe the associated cell complex. We 
begin by recalling the original construction in some detail; there is nothing new in our presentation, except maybe that we make an effort to be gentle to non-topologists. After lifting this construction, we will prove the following theorem:

Theorem 3.1 Let $\Sigma$ be a closed Riemann surface of genus $g$. Let $\widetilde{\operatorname{Sym}^{\infty}(\Sigma)}$ be the space

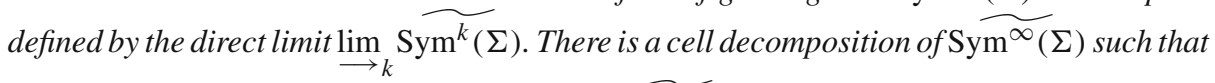
the corresponding cellular chain complex $\mathcal{C}_{*}\left(\widetilde{\operatorname{Sym}^{\infty}(\Sigma)}\right)$ is the differential graded algebra

$$
\mathbb{Z}\left[x_{1}, \ldots x_{g}, y_{1}, \ldots y_{g}\right] \otimes \Lambda\left[e_{1}, \ldots, e_{g}, f_{1}, \ldots f_{g}\right] \otimes \Gamma\left[\gamma_{2}\right] .
$$

The degrees of the generators $x_{i}, y_{i}$ are 0 , the degrees of the generators $e_{i}, f_{i}$ are 1 , and the degree of $\gamma_{s}$ is $s$. The boundary map is given by

$$
\begin{aligned}
& \partial\left(e_{i}\right)=1-x_{i} \\
& \partial\left(f_{i}\right)=1-y_{i}, \\
& \partial\left(\gamma_{s}\right)=\sum_{i}\left(\left(1-y_{i}\right) e_{i}-\left(1-x_{i}\right) f_{i}\right) \gamma_{s-2} .
\end{aligned}
$$

This cell decomposition restricts to cell decompositions of each $k$-skeleton $\operatorname{Sk}^{k}\left(\widetilde{\operatorname{Sym}^{\infty}(\Sigma)}\right)=$ $\widetilde{\operatorname{Sym}^{k}(\Sigma)}$. Give $\mathcal{C}_{*}\left(\widetilde{\operatorname{Sym}^{\infty}(\Sigma)}\right)$ a multiplicative grading $\mathcal{F}$ such that the gradings of $e_{i}$ and $f_{i}$ equal 1, and the grading of $\gamma_{s}$ equals $s / 2$. Then the cellular chain complex of $\operatorname{Sk}^{k}(\Sigma)$ equals $\oplus_{i \leq k} \mathcal{F}^{i} \mathcal{C}_{*}\left(\widehat{\operatorname{Sym}^{\infty}(\Sigma)}\right)$.

The more technical algebraic terms used in the statement of this theorem will be clarified in the subsections below.

\subsection{Recollection of a construction by Kallel and Salvatore}

This is a general construction which for any two-dimensional CW-complex $Y$ and any natural number $k$ produces a CW-complex $\overline{\operatorname{Sym}^{k}(Y)}$, together with a homotopy equivalence $r$ : $\operatorname{Sym}^{k}(Y) \rightarrow \overline{\operatorname{Sym}^{k}(Y)}$. It uses special properties of cells of dimensions one and two, and the arguments we shall present do not seem to have an obvious direct generalisation to higher dimensions.

We will mainly be interested in the special case where $Y$ is a cell decomposition of a closed oriented surface $\Sigma$ with one 0 -cell, $2 g$ 1-cells and one 2-cell. It is convenient to do the construction in two steps, first treating the 1-skeleton of the surface. We will denote this 1 -skeleton by $X$, so that

$$
X:=\mathrm{Sk}^{1} Y \simeq \Sigma \backslash\{\mathrm{pt}\} .
$$

Thus $X$ is a one-dimensional CW-complex with one 0 -cell. Chose the basepoint $v_{0} \in X$ to be the point it represents.

The 1-cells of $X$ come with characteristic maps $\phi_{i}: D_{i}^{1} \rightarrow X$. We pick a basepoint in the boundary of each $D^{1}$. All attaching maps are automatically basepoint-preserving. The $k$-fold product $X^{k}$ has a corresponding cell decomposition; the cells are indexed by sequences $I=\left(i_{1}, \ldots, i_{k}\right)$, where each $i_{j}$ is the index of a 1 -cell in $X$. Let $D^{I}=D^{k}$. The characteristic maps of the cells are just products

$$
\phi_{I}=\prod_{1 \leq j \leq k} \phi_{i_{j}}: D^{I} \cong \prod_{1 \leq j \leq k} D^{1} \rightarrow X^{k} .
$$


These characteristic maps are also basepoint-preserving. The $s$-skeleton of $X^{k}$ is built inductively by attaching $s$-cells to the $(s-1)$-skeleton:

$$
\operatorname{Sk}^{s}\left(X^{k}\right)=\operatorname{Sk}^{s-1}\left(X^{k}\right) \cup_{\phi_{I}}\left(\cup_{I} D^{I}\right) .
$$

The action of the symmetric group $\mathfrak{S}_{k}$ on $X^{k}$ preserves the $s$-skeleton. We obtain a decomposition

$$
\operatorname{Sk}^{s}\left(X^{k}\right) / \mathfrak{S}_{k}=\left(X^{k-1} / \mathfrak{S}_{k}\right) \cup_{\phi}\left(\cup_{I} D^{I} / \mathfrak{S}_{k}\right) .
$$

A free orbit of cells in $X^{k}$ under $\mathfrak{S}_{k}$ will contribute a single cell to this pushout. More generally, if a cell in $X^{k}$ is built from $l$ times the 0 -cell, and $k-l$ distinct 1 -cells, its $\mathfrak{S}_{k}$-orbit will consist of $\left(\begin{array}{l}k \\ l\end{array}\right)$ cells. Each open cell in this orbit will map homeomorphically to $\operatorname{Sk}^{s}\left(X^{k}\right) / \mathfrak{S}_{k}$. The union of the corresponding closed cells forms a subspace $\overline{\operatorname{Sym}^{k}(X)} \subset \operatorname{Sym}^{k}(X)$, which has a CW-structure, with the cells we have just described. The inclusion of this subspace is a homotopy equivalence, and there exists a retraction (which is also a homotopy equivalence)

$$
r: \operatorname{Sym}^{k}(X) \rightarrow \overline{\operatorname{Sym}^{k}(X)}
$$

This completes the construction for a 1-dimensional CW-complex $X$ with a single 0 -cell. The next step is to extend the construction to the case when the CW-complex also has 2-cells. Actually, as we have already said, in this paper we are only interested in the cell structure on a closed surface $\Sigma$, formed by gluing a single 2-cell onto a bouquet of circles, and we will specialize to this later.

We have to produce characteristic maps of cells. To generate such maps, we use concatenation, which is the obvious map

$$
\mu_{k, l}: \operatorname{Sym}^{k}(X) \times \operatorname{Sym}^{l}(X) \rightarrow \operatorname{Sym}^{k+l}(X),
$$

and which we also write as $\mu(x, y)=x * y$. As in the 1-dimensional case, we do not give the symmetric product itself a cell structure, but we produce a cell complex which is homotopy equivalent to the symmetric product.

Following [31], given a 2-complex $Y$ whose 1-skeleton $X=\mathrm{Sk}^{1} Y$ is a CW-complex with a single 0-cell, we define $\overline{\operatorname{Sym}^{k}(Y)}$ as the quotient of $\operatorname{Sym}^{k}(Y)$ which for $x, x^{\prime} \in \underline{\operatorname{Sym}^{l}(X)}$ and $y \in \operatorname{Sym}^{k-l}(Y)$ identifies $x * y$ with $x^{\prime} * y$, if $x$ and $x^{\prime}$ have the same image in $\overline{\operatorname{Sym}^{l}(X)}$. The quotient map

$$
r: \operatorname{Sym}^{k}(Y) \rightarrow \overline{\operatorname{Sym}^{k}(Y)}
$$

is a homotopy equivalence. In this case, there is no corresponding inclusion of $\overline{\operatorname{Sym}^{k}(Y)}$ into $\operatorname{Sym}^{k}(Y)$, so $r$ is not a deformation retraction to a subspace.

The retraction (12) is compatible with concatenation (11), so there are commutative diagrams

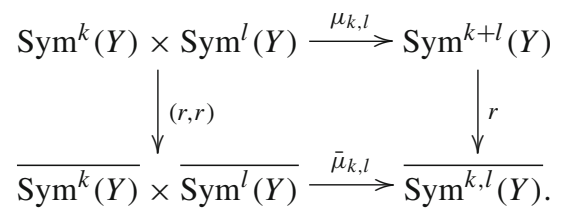

The attaching maps of cells are given in the following fashion. The cells in $\overline{\operatorname{Sym}^{k}(X)}=$ $\overline{\operatorname{Sym}^{k}\left(\mathrm{Sk}^{1} Y\right)}$ are products of distinct 1-cells

$$
D^{k} \cong\left(D^{1}\right)^{k} \rightarrow X^{k}=\left(\operatorname{Sym}^{1}(X)\right)^{k} \stackrel{\mu}{\rightarrow} \operatorname{Sym}^{k}(X) \stackrel{r}{\rightarrow} \overline{\operatorname{Sym}^{k}(X)} .
$$


There is also the 2-cell in $Y=X \cup e^{2}$ with characteristic map $\gamma_{2}: D^{2} \rightarrow Y$. We can assume that $\gamma_{2}$ is basepoint-preserving. Using concatenation, it produces cells of all even dimensions

$$
\gamma_{2 j}: D^{2 j} \cong\left(D^{2}\right)^{j} \stackrel{\left(\gamma_{2}\right)^{j}}{\rightarrow} Y^{k} \stackrel{\mu}{\rightarrow} Y .
$$

Finally, we can concatenate the cells in $\overline{\operatorname{Sym}^{k-j}(X)}$ with $\gamma_{j}$ for $1 \leq j \leq g$. This produces a complete list of cells in a cell decomposition of $\overline{\operatorname{Sym}^{k}(Y)}$. Note that every characteristic map is basepoint-preserving. The homology of $\overline{\operatorname{Sym}^{k}(Y)}$ can be computed as the homology of the corresponding cellular chain complex $C_{*}\left(\overline{\operatorname{Sym}^{k}(Y)}\right)$, whose chain group $C_{n}\left(\overline{\operatorname{Sym}^{k}(Y)}\right)$ has a basis indexed by the $n$-cells (see for instance Chapter 2.2 of [24] for the definition of chain complexes and a discussion of cellular homology).

We note that concatenation induces a commutative product

$$
\overline{\mu_{k, l}}: \overline{\operatorname{Sym}^{k}(Y)} \times \overline{\operatorname{Sym}^{l}(Y)} \rightarrow \overline{\operatorname{Sym}^{k+l}(Y)} .
$$

In particular, multiplication with the basepoint $v_{0} \in \overline{\operatorname{Sym}^{k}(Y)}$ induces stabilization maps $i: \overline{\operatorname{Sym}^{k}(Y)} \rightarrow \overline{\operatorname{Sym}^{k+l}(Y)}$. By the description of the cells of $\overline{\operatorname{Sym}^{k}(Y)}$, this map identifies $\overline{\operatorname{Sym}^{k}(Y)}$ with a subcomplex of $\overline{\operatorname{Sym}^{k+l}(Y)}$. It follows that the induced map of chain groups $i_{*}: C_{*}\left(\overline{\operatorname{Sym}^{k}(Y)}\right) \rightarrow C_{*}\left(\overline{\operatorname{Sym}^{k+l}(Y)}\right)$ is a split injective map.

The multiplication passes to a graded commutative product of chain groups

$$
\overline{\mu_{k, l *}}: C_{m}\left(\overline{\operatorname{Sym}^{k}(\Sigma)}\right) \otimes C_{n}\left(\overline{\operatorname{Sym}^{l}(\Sigma)}\right) \rightarrow C_{m+n}\left(\overline{\operatorname{Sym}^{k+l}(\Sigma)}\right) .
$$

Here, graded commutative means that $\mu_{k, l *}(a \otimes b)=(-1)^{m n} \mu_{l, k *}(b \otimes a)$. Moreover, the differential in the cellular chain complex is a derivation with respect to this product. That is, if $a \in C_{m}\left(\overline{\operatorname{Sym}^{k}(Y)}\right)$ and $b \in C_{n}\left(\overline{\operatorname{Sym}^{l}(Y)}\right)$, then $\partial \mu_{k, l *}(a \otimes b)=\mu_{k, l *}(\partial a \otimes b)+$ $(-1)^{k} \mu_{k, l *}(a \otimes \partial b)$.

This product exhibits $\lim _{k} C_{*}\left(\overline{\operatorname{Sym}^{k}(Y)}\right)$ as a graded commutative ring, containing $C_{*}\left(\overline{\operatorname{Sym}^{k}(Y)}\right)$ as a direct summand. Note that this summand is not closed under ring multiplication. Since the boundary map is a derivation, $\lim _{k} C_{*}\left(\overline{\operatorname{Sym}^{k}(Y)}\right)$ is a differential graded algebra (DGA), i.e. a graded algebra together with a chain complex structure whose differential preserves the algebraic structure (see [21], Chapter V.3).

Example 3.2 If $S^{1}$ is the $\mathrm{CW}$-complex with one 0 -cell and one 1 -cell $e_{1}$, then for any $k \geq 1$ the stabilization map

$$
S^{1}=\overline{\operatorname{Sym}^{1}\left(S^{1}\right)} \rightarrow \overline{\operatorname{Sym}^{k}\left(S^{1}\right)}
$$

is a homeomorphism onto its image. The $\operatorname{ring} \lim _{k} C_{*}\left(\overline{\operatorname{Sym}^{k}\left(S^{1}\right)}\right)$ equals the exterior algebra $\Lambda\left[e_{1}\right]=\mathbb{Z}\left[e_{1}\right] / e_{1}^{2}=0$. The differential is trivial, and for $k \geq 1$ the inclusion map $C_{*}\left(\overline{\operatorname{Sym}^{k}\left(S^{1}\right)}\right) \subset \underset{\lim _{k}}{ } C_{*}\left(\overline{\operatorname{Sym}^{k}\left(S^{1}\right)}\right)$ is an isomorphism.

Example 3.3 Let $X$ be a 1-dimensional CW-complex with one 0 -cell and 1-cells $e_{1}, \ldots, e_{n}$. The inclusion of the cells collectively define an isomorphism $\Lambda\left[e_{1}, \ldots, e_{n}\right] \rightarrow$ $\lim _{k} C_{*}\left(\overline{\operatorname{Sym}^{k}(X)}\right)$. The differential is trivial, and under this isomorphism the image of the inclusion $C_{*}\left(\overline{\operatorname{Sym}^{k}(X)}\right) \hookrightarrow \lim _{\longrightarrow} C_{*}\left(\overline{\operatorname{Sym}^{k}(X)}\right)$ consists of monomials without repetitions in the generators with $k$ or less factors. In particular, if $k \geq n$, this inclusion is an isomorphism. 
We now specialize to the case we really are interested in. So let $Y$ be a cell decomposition of a surface $\Sigma_{g}$ of genus $g$, with one 0 -cell $v_{0}, 2 g$ 1-cells and one 2-cell. We choose the 1-cells in sets of pairs $e_{i}, f_{i}, 1 \leq i \leq g$, and let the 2-cell $D^{2}$ be attached by the word $\prod_{1 \leq i \leq g}\left(e_{i} f_{i} e_{i}^{-1} f_{i}^{-1}\right)$. We can assume that the loop $e_{i}$ represents $x_{i}$ (considered as class in $H_{1}(\Sigma ; \mathbb{Z})$ ) and that the loop $f_{i}$ represents $y_{i}$ (see Sect. 2). We also chose a basepoint in the boundary of each standard disc $D^{n}(n=1,2)$.

Let $\gamma_{2}: D^{2} \rightarrow Y \rightarrow \lim _{k} C_{*}\left(\overline{\operatorname{Sym}^{k}(X)}\right)$ be the characteristic map of the 2-cell. Taking powers of this map, and dividing by the action of the symmetric group, we obtain maps

$$
\gamma_{2 n}: D^{2 n} \cong\left(D^{2}\right)^{k} \stackrel{\gamma_{2}^{n}}{\longrightarrow} \overline{\operatorname{Sym}^{n}(X)} .
$$

We abusively denote the corresponding cycles by $\gamma_{2 n}$.

According to Example 3.3, the inclusion $X=\operatorname{Sk}^{1}(Y) \subset Y$ induces an inclusion

$$
\Lambda\left[e_{1}, \ldots, e_{g}, f_{1}, \ldots, f_{g}\right] \hookrightarrow \lim _{k} C_{*}\left(\overline{\operatorname{Sym}^{k}(X)}\right) .
$$

This makes $\lim _{k} C_{*}\left(\overline{\operatorname{Sym}^{k}(Y)}\right)$ into a free module over $\Lambda\left[e_{1}, \ldots, e_{g}, f_{1}, \ldots, f_{g}\right]$ with generators $\gamma_{2 n}, \overrightarrow{n \geq 0} 0$. The symmetrising map

$$
\left(D^{2}\right)^{k} \rightarrow\left(D^{2}\right)^{k} / \mathfrak{S}_{k} \cong D^{2 k}
$$

has degree $k$ !. It follows that $\left(\gamma_{2}\right)^{k}=k ! \gamma_{2 k}$, and consequently that

$$
\gamma_{2 k} \gamma_{2 l}=\left(\begin{array}{c}
k+l \\
k
\end{array}\right) \gamma_{2(k+l)}
$$

The relation (13) means that the characteristic map $\gamma_{2}$ of the 2-cell generates a subalgebra in the cellular chain complex that is isomorphic to what is called a gamma-algebra, and which we will denote by $\Gamma\left[\gamma_{2}\right]$ following general practice. More concretely, as a group $\Gamma\left[\gamma_{2}\right]$ is the free Abelian group generated by the classes $\gamma_{2 n}$ for $n \in \mathbb{N}_{0}$. This comes with a grading, generated additively from the prescription that $\gamma_{2}$ has degree two; so $\gamma_{2 n}$ has degree $2 n$. The multiplication is defined on generators exactly by the relation (13).

It follows from the definitions that

$$
\lim _{k} C_{*}\left(\overline{\operatorname{Sym}^{k}(Y)}\right) \cong \Lambda\left[e_{1}, \ldots e_{g}, f_{1}, \ldots, f_{g}\right] \otimes \Gamma\left[\gamma_{2}\right] .
$$

The differential on $e_{1}$ is trivial by naturality. Since $\gamma_{2}$ is the top cell in $\Sigma_{g}$, the differential on $\gamma_{2}$ is zero. It follows from the Leibniz rule that $\partial\left(k ! \gamma_{2} k\right)=\partial\left(\gamma_{2}^{k}\right)=k !\left(\gamma_{2}\right)^{k-1} \partial \gamma_{2}=0$. Since the chain groups are free groups, it follows that $\partial \gamma_{k}=0$, and thus that the differential is trivial. If we introduce the grading $\operatorname{deg}\left(e_{i}\right)=\operatorname{deg}\left(f_{j}\right)=1, \operatorname{deg}\left(\gamma_{i}\right)=i$, the image $C_{*}\left(\overline{\operatorname{Sym}^{k}(Y)}\right) \subset \lim _{k} C_{*}\left(\overline{\operatorname{Sym}^{k}(Y)}\right)$ exactly consists of the classes in grading $\leq k$.

Because the differential is trivial, the chain groups agree with the homology groups. This completes the computation in [31] of the homology of $\operatorname{Sym}^{k}(\Sigma)$.

\subsection{The chain complex of the universal covering space}

We now consider the universal cover of $\operatorname{Sym}^{k}(Y)$ for $k \geq 2$. As before, fix a standard basis $\left\{x_{1}, \ldots, x_{g}, y_{1}, \ldots y_{g}\right\}$ as in (7) for

$$
\pi:=\pi_{1}\left(\operatorname{Sym}^{k}(\Sigma)\right) \cong H_{1}(\Sigma ; \mathbb{Z}) .
$$


The cell decomposition of $\overline{\operatorname{Sym}^{k}(Y)}$ studied in the previous subsection lifts to a cell decomposition of the universal cover

$$
U_{k}(Y):=\widetilde{\operatorname{Sym}^{k}(Y)}
$$

for $k \geq 2$. Each cell of $\overline{\operatorname{Sym}^{k}(Y)}$ lifts to a free $\pi$-orbit of cells in the universal cover. In particular, the basepoint $v_{0} \in \overline{\operatorname{Sym}^{k}(Y)}$ is covered by a free $\pi$-orbit of points. Pick one of the points in this orbit to be the basepoint of $U_{k}(Y)$.

For $k \in\{0,1\}$ we similarly consider the covering $U_{k}(Y)$ of $\overline{\operatorname{Sym}^{k}(Y)}$ corresponding to $\pi$. So $U_{0}(Y)$ equals $\pi$ as a discrete set, and $U_{1}(Y)$ is the simply connected covering of $Y$ determined by the kernel of the Hurewicz map. Note that there is a short exact sequence of monoids:

$$
\pi \rightarrow \coprod_{k \geq 0} U_{k}(Y) \rightarrow \coprod_{k \geq 0} \overline{\operatorname{Sym}^{k}(Y)}
$$

We lift the basepoint to $0 \in \pi$. The cells of $\overline{\operatorname{Sym}^{k}(Y)}$ lift to cells of $U_{n}(Y)$. More precisely, the characteristic maps of the cells in $\overline{\operatorname{Sym}^{k}(Y)}$ lifts to character maps of cells in $U_{n}(Y)$.

Recall that each cell of positive dimension contains the basepoint in its boundary, and there is a unique basepoint preserving lifting of the characteristic map. By abuse of notation, we use the same notation for a based cell and its based lifting. We obtain all cells in $U_{n}(Y)$ by translating these cells by the elements of $\pi$. It follows that we have a CW-structure on $U_{n}(\Sigma)$ with cells

$$
\alpha * v_{0}^{k} * e_{i_{1}} * \cdots * e_{i_{l}} * f_{j_{1}} * \cdots f_{j_{m}} * \gamma_{s},
$$

where $\alpha \in \pi$ and $k+j+m+s=n$. The cellular chain groups are

$$
\mathbb{Z}\left[x_{1}^{ \pm 1}, \ldots x_{g}^{ \pm 1}, y_{1}^{ \pm 1}, \ldots y_{g}^{ \pm 1}\right] \otimes \Lambda\left[e_{1}, \ldots, e_{g}, f_{1}, \ldots, f_{g}\right] \otimes \Gamma\left[\gamma_{2}\right] .
$$

The differentials are derivations. Since $e_{i}$ is the path representing $x_{i}$, we have that $\partial e_{i}=x_{i}-1$, and similarly $\partial f_{i}=y_{i}-1$. The differential of $\gamma_{2}$ is given by tracing the boundary of the lifted cell. For concreteness, we draw the lifted cell for the particular case of a genus two surface including the names of all cells in its boundary. The 0 -cell $g v_{0}$ is denoted by the corresponding group element $g$ :

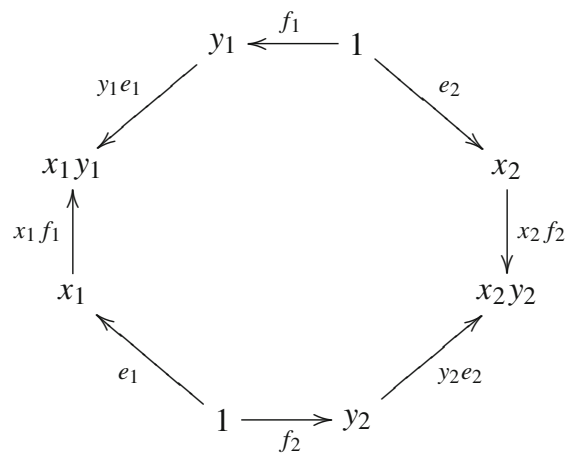

We obtain that

$$
\partial \gamma_{2}=\sum_{i}\left(e_{i}+x_{i} f_{i}-y_{i} e_{i}-f_{i}\right)=\sum_{i}\left(\left(1-y_{i}\right) e_{i}-\left(1-x_{i}\right) f_{i}\right),
$$


and since $\partial$ is a derivation, that

$$
\partial \gamma_{s}=\sum_{i}\left(\left(1-y_{i}\right) e_{i}-\left(1-x_{i}\right) f_{i}\right) \gamma_{s-2}
$$

Let us write $\lambda:=\sum_{i}\left(\left(1-y_{i}\right) e_{i}-\left(1-x_{i}\right) f_{i}\right)$, so that this equation simplifies to

$$
\partial \gamma_{s}=\lambda \gamma_{s-2}
$$

\section{Homology computations}

In this section and the next, $X_{n}$ will denote a one-dimensional CW-complex with $n$ onecells; $Y_{g}$ will denote a two-dimensional complex $Y_{g}:=X_{2 g} \cup e_{2}$ such that $Y_{g}$ is homotopy equivalent to the closed Riemann surface of genus $g$ that we call $\Sigma_{g}$. That is, $Y_{g}$ is a choice of a CW-structure on $\Sigma_{g}$.

The universal cover of the symmetric product $\operatorname{Sym}^{k}(\Sigma)$ which we denote $\widetilde{\operatorname{Sym}^{k}\left(\Sigma_{g}\right)}$, is homotopy equivalent to the universal cover of $\overline{\operatorname{Sym}^{k}\left(Y_{g}\right)}$. Restricting this universal cover to the symmetric product of the one skeleton $X_{2 g}$ of $Y_{g}$, we obtain a (not universal) covering space $U_{k}\left(X_{2 g}\right)$ of $\overline{\operatorname{Sym}^{k}\left(X_{2 g}\right)}$. The total space of this cover has a CW-structure as a subcomplex of $U_{k}\left(Y_{g}\right)$. In Theorem 3.1 we used this CW-structure to determine a chain complex whose homology equals the homology of $U_{k}\left(Y_{g}\right)$. In this section, we shall compute the homology of that chain complex.

The strategy of the computation will be as follows. We first compute the homology of $U_{k}\left(X_{2 g}\right)$; this is done in Lemma 4.3. Then we relate $H_{*}\left(U_{k}\left(Y_{g}\right)\right)$ to the homology of $U_{k}\left(X_{2 g}\right)$ in Lemma 4.5. In order to complete the calculation, we have to do some homological algebra. The main step of this homological calculation is collected in Lemma 4.6, before stating and proving our main Theorem 4.7. (This calculation can also be formulated using spectral sequences, but we prefer to keep the formal machinery as elementary as we can.)

Let $X=X_{n}$ be a wedge of $n$ copies of the circle. The cellular homology complex corresponding to the cell decomposition of the symmetric product that we discussed in Sect. 3 can, as explained there, be identified with the DGA

$$
\mathcal{C}_{*}(X)=\mathbb{Z}\left[z_{1}^{ \pm 1}, \ldots, z_{n}^{ \pm 1}\right] \otimes \Lambda\left[\psi_{1}, \ldots, \psi_{n}\right], \quad \partial \psi_{i}=z_{i}-1 .
$$

Here $\mathbb{Z}\left[z_{1}^{ \pm}, \ldots z_{n}^{ \pm}\right]=\mathbb{Z}[\pi]$, and $\Lambda\left[\psi_{1}, \ldots, \psi_{n}\right]$ is the exterior algebra on the generators $\psi_{i}$, each of degree 1 .

This chain complex computes the homology of $\lim _{k} C_{*}\left(U_{k}\left(X_{n}\right)\right)$. To obtain the homology of $C_{*}\left(U_{k}\left(X_{n}\right)\right.$ ), we have to take the subcomplex corresponding to classes of grading $\leq k$. Fortunately, the grading agrees with the internal grading, so the subcomplex $\mathcal{C}_{*}\left(U_{k}\left(X_{n}\right)\right) \subset$ $\mathcal{C}_{*}\left(X_{n}\right)$ is the truncated complex

$$
\mathcal{C}_{k}\left(X_{n}\right) \stackrel{\partial_{k}}{\rightarrow} \cdots \mathcal{C}_{1}\left(X_{n}\right) \stackrel{\partial_{1}}{\rightarrow} \mathcal{C}_{0}\left(X_{n}\right)
$$

We will use this complex to compute the homology of $U_{k}\left(X_{n}\right)$.

Definition 4.1 $\mathbf{K}_{k}:=\operatorname{ker}\left\{\partial_{k}: \mathcal{C}_{k}\left(X_{n}\right) \rightarrow \mathcal{C}_{k-1}\left(X_{n}\right)\right\}$.

Remark 4.2 If $0 \leq k<n, \mathbf{K}_{k}$ is not finitely generated as an Abelian group. In fact, $\mathcal{C}_{k}\left(X_{n}\right)$ contains no $\mathbb{Z}[\pi]$-torsion, so that any nontrivial element of $\mathbf{K}_{k} \subset \mathcal{C}_{k}\left(X_{n}\right)$ generates a free 
$\mathbb{Z}[\pi]$-submodule of rank one. As an Abelian group, this submodule is free on infinitely many generators. An example of a nontrivial element in $\mathbf{K}_{k}$ for $0 \leq k<n$ is $\partial\left(\psi_{1} \psi_{2} \cdots \psi_{k+1}\right)$.

Lemma 4.3 Assume that $k \geq 2$.

$$
H_{i}\left(U_{k}\left(X_{n}\right)\right)= \begin{cases}\mathbb{Z} & \text { if } i=0 \\ \mathbf{K}_{k} & \text { if } 0<i=k \leq n \\ 0 & \text { else }\end{cases}
$$

Proof The untruncated chain complex

$$
0 \rightarrow \mathcal{C}_{n}\left(X_{n}\right) \stackrel{\partial_{k}}{\rightarrow} \ldots \mathcal{C}_{1}\left(X_{n}\right) \stackrel{\partial_{1}}{\rightarrow} \mathcal{C}_{0}\left(X_{n}\right) \rightarrow 0
$$

is the tensor product of $n$ copies of the chain complex

$$
\mathbb{Z}\left[z^{ \pm 1}\right] \otimes \Lambda[\psi], \quad \partial \psi=z-1 .
$$

The homology of this chain complex is $\mathbb{Z}$, generated by the class 1 in dimension 0 , so by Künneth's formula (see [34] Chapter V, Theorem 10.1), the homology of $\mathcal{C}_{*}\left(X_{n}\right)$ also consists of $\mathbb{Z}$ in dimension 0 . The lemma follows on comparing this with the homology of the truncated chain complex.

The cellular chain complex $\mathcal{C}_{*}^{k}\left(Y_{g}\right)$ of $U_{k}\left(Y_{g}\right)$ is the subcomplex of

$$
\Gamma\left[\gamma_{2}\right] \otimes \mathbb{Z}\left[x_{1}^{ \pm 1}, \ldots x_{g}^{ \pm 1}, y_{1}^{ \pm 1}, \ldots y_{g}^{ \pm 1}\right] \otimes \Lambda\left[e_{1}, \ldots, e_{g}, f_{1}, \ldots, f_{g}\right]=\Gamma\left[\gamma_{2}\right] \otimes \mathcal{C}_{*}\left(X_{2 g}\right)
$$

generated by cells of grading $\leq k$, where the grading is multiplicative on the cells, the gradings of $x_{i}$ and $y_{i}$ are zero, the gradings of $e_{i}$ and $f_{i}$ are 1 , and the grading of each $\gamma_{2 i}$ is $i$. This time, the grading does not agree with the internal grading because the internal grading of $\gamma_{2 i}$ is $2 i$, not $i$.

There is a natural chain of CW-complexes

$$
\{\mathrm{pt}\}=U_{0}\left(\Sigma_{g}\right) \subset U_{1}\left(Y_{g}\right) \subset U_{2}\left(Y_{g}\right) \subset \cdots \subset U_{k}\left(Y_{g}\right) \subset \cdots
$$

Each inclusion is the inclusion of a subcomplex. There is a corresponding sequence of cellular chain complexes. Let $Q_{k}=\mathcal{C}_{*} U_{k}\left(Y_{g}\right) / \mathcal{C}_{*} U_{k-1}\left(Y_{g}\right)$ be the quotient chain complex. We consider $\mathcal{C}_{*}\left(X_{2 g}\right)$ as a subcomplex of $\mathcal{C}_{*}\left(Y_{g}\right)$, using the identifications $x_{i}=\psi_{i}, y_{j}=\psi_{g+j}$ for $1 \leq i, j \leq g$.

Lemma 4.4 The homology of the chain complex $Q_{k}$ is concentrated in one dimension. More precisely

$$
H_{i}\left(Q_{k}\right) \cong \begin{cases}\mathcal{C}_{k}\left(X_{2 g}\right) / \lambda \mathcal{C}_{k}\left(X_{2 g}\right) & \text { if } k \leq 2 g \text { and } i=k \\ \mathbb{Z} \text { generated by } \gamma_{2 k-2 g} & \text { if } k \geq 2 g \text { and } i=2 k-2 g \\ 0 & \text { else. }\end{cases}
$$

Proof The chain complex $Q_{k}$ is given by

$$
0 \rightarrow \gamma_{2 k} \mathcal{C}_{0}\left(X_{2 g}\right) \stackrel{\partial}{\rightarrow} \gamma_{2 k-2} \mathcal{C}_{1}\left(X_{2 g}\right) \stackrel{\partial}{\rightarrow} \cdots \stackrel{\partial}{\rightarrow} \gamma_{2} \mathcal{C}_{k-1}\left(X_{2 g}\right) \stackrel{\partial}{\rightarrow} \mathcal{C}_{k}\left(X_{2 g}\right) \rightarrow 0 .
$$

Because $\partial \gamma_{i}=\lambda \gamma_{i-2}$, this chain complex is isomorphic to the chain complex

$$
0 \rightarrow \mathcal{C}_{0}\left(X_{2 g}\right) \stackrel{\lambda}{\rightarrow} \mathcal{C}_{1}\left(X_{2 g}\right) \stackrel{\lambda}{\rightarrow} \cdots \stackrel{\lambda}{\rightarrow} \mathcal{C}_{k-1}\left(X_{2 g}\right) \stackrel{\lambda}{\rightarrow} \mathcal{C}_{k}\left(X_{2 g}\right) \rightarrow 0 .
$$


We first consider the corresponding complex

$$
0 \rightarrow \mathcal{C}_{0}\left(X_{2 g}\right) \stackrel{\lambda}{\rightarrow} \mathcal{C}_{1}\left(X_{2 g}\right) \stackrel{\lambda}{\rightarrow} \ldots \mathcal{C}_{2 g-1}\left(X_{2 g}\right) \stackrel{\lambda}{\rightarrow} \mathcal{C}_{2 g}\left(X_{2 g}\right) \rightarrow 0,
$$

and claim that its cohomology is 0 except in degree $2 g$, where the cohomology is $\mathbb{Z}$.

We can write the complex $\mathcal{C}_{*}\left(X_{2 g}\right)$ as a tensor product

$$
\bigotimes_{i=1}^{g}\left(\mathbb{Z}\left[x_{i}^{ \pm 1}\right] \otimes \Lambda\left[f_{i}\right]\right) \otimes \bigotimes_{j=1}^{g}\left(\mathbb{Z}\left[y_{j}^{ \pm 1}\right] \otimes \Lambda\left[e_{j}\right]\right)
$$

where the boundary maps are multiplication by $-\left(1-x_{i}\right) f_{i}$ in $\mathbb{Z}\left[x_{i}^{ \pm 1}\right] \otimes \Lambda\left[f_{i}\right]$, respectively multiplication by $\left(1-y_{j}\right) e_{j}$ in $\mathbb{Z}\left[y_{j}^{ \pm 1}\right] \otimes \Lambda\left[e_{j}\right]$. The cohomology of $\mathbb{Z}\left[x_{i}^{ \pm 1}\right] \otimes \Lambda\left[f_{i}\right]$ is a copy of $\mathbb{Z}$ generated by $f_{i}$, and the cohomology of $\mathbb{Z}\left[y_{i}^{ \pm 1}\right] \otimes \Lambda\left[e_{i}\right]$ is a copy of $\mathbb{Z}$ generated by $e_{i}$; actually, there is a short exact sequence

$$
\begin{aligned}
0 \rightarrow \mathbb{Z}\left[x_{i}^{ \pm 1}\right] \stackrel{\left(x_{i}-1\right) f_{i}}{\longrightarrow} f_{i} \mathbb{Z}\left[x_{i}^{ \pm 1}\right] & \rightarrow \mathbb{Z} \rightarrow 0 . \\
f_{i} \sum_{k} n_{k} x_{i}^{k} & \mapsto \sum_{k} n_{k}
\end{aligned}
$$

It follows from the Künneth formula that the cohomology of $\mathcal{C}_{*}$ is a single copy of $\mathbb{Z}$ generated by $\left(\prod_{1 \leq j \leq g} e_{j}\right)\left(\prod_{1 \leq i \leq g} f_{i}\right)$.

We now return to the truncated chain complex (15). This chain complex is exact, except at $\mathcal{C}_{k}\left(X_{2 g}\right)$ if $k<2 g$ and at $\mathcal{C}_{2 g}\left(X_{2 g}\right)$ if $k \geq 2 g$. The lemma follows.

\section{Lemma 4.5 Let}

$$
\partial_{k}: \mathcal{C}_{k}\left(X_{2 g}\right) / \lambda \mathcal{C}_{k-1}\left(X_{2 g}\right) \rightarrow \mathcal{C}_{k-1}\left(X_{2 g}\right) / \lambda \mathcal{C}_{k-2}\left(X_{2 g}\right)
$$

be the boundary map.

$$
H_{i}\left(U_{k}\left(Y_{g}\right)\right) \cong \begin{cases}\mathbb{Z} & \text { if } i \text { is even, } k>2 g \text { and } i \leq 2 k-2 g, \\ \operatorname{ker} \partial_{k} & \text { if } i=k \text { and } k \leq 2 g \\ \operatorname{ker} \partial_{i} / \operatorname{im} \partial_{i-1} & \text { if } i<k \leq 2 g . \\ 0 & \text { else. }\end{cases}
$$

Proof The long exact sequence

$$
\cdots \rightarrow H_{i}\left(\mathcal{C}_{*} U_{k-1}\left(Y_{g}\right)\right) \rightarrow H_{i}\left(\mathcal{C}_{*} U_{k}\left(Y_{g}\right)\right) \rightarrow H_{i}\left(Q_{k}\right) \rightarrow \cdots
$$

breaks up into shorter exact sequences, since $H_{i}\left(Q_{k}\right)$ is only nontrivial in a single dimension. We need to consider several cases separately.

When $k \leq 2 g$, we have isomorphisms

$$
H_{i}\left(\mathcal{C}_{*} U_{k-1}\left(Y_{g}\right)\right) \rightarrow H_{i}\left(\mathcal{C}_{*} U_{k}\left(Y_{g}\right)\right), \quad i \neq k-1, k,
$$

together with the exact sequence

$$
\begin{gathered}
0 \longrightarrow H_{k}\left(\mathcal{C}_{*} U_{k-1}\left(Y_{g}\right)\right) \longrightarrow H_{k}\left(\mathcal{C}_{*} U_{k}\left(Y_{g}\right)\right) \longrightarrow H_{k}\left(Q_{k}\right) \\
\longrightarrow H_{k-1}\left(\mathcal{C}_{*} U_{k-1}\left(Y_{g}\right)\right) \longrightarrow H_{k-1}\left(\mathcal{C}_{*} U_{k}\left(Y_{g}\right)\right) \longrightarrow
\end{gathered}
$$


(I.1) The case $k \leq 2 g$ and $i>k$ : If $i>2 k$, then $\mathcal{C}_{i}\left(U_{k}\left(Y_{g}\right)\right)=0$, so certainly $H_{i}\left(\mathcal{C}_{*} U_{k}\left(Y_{g}\right)\right)=0$. It follows by downwards induction from this and from (16) that if $k \leq 2 g$ and $i>k$, then $H_{i}\left(\mathcal{C}_{*} U_{k}\left(Y_{g}\right)\right)=0$.

(I.2) The case $k \leq 2 g$ and $i=k$ : It follows from case (I.1) that $H_{k}\left(\mathcal{C}_{*} U_{k-1}\left(Y_{g}\right)\right) \cong 0$, so that by (17) the group $H_{k}\left(\mathcal{C}_{*} U_{k}\left(Y_{g}\right)\right)$ is isomorphic to the kernel of the map $H_{k}\left(Q_{k}\right) \rightarrow$ $H_{k-1}\left(\mathcal{C}_{*} U_{k-1}\left(Y_{g}\right)\right)$.

Moreover, it also follows from (17) that $H_{k}\left(\mathcal{C}_{*} U_{k}\left(Y_{g}\right)\right) \rightarrow H_{k}\left(Q_{k}\right)$ and similarly $H_{k-1}\left(\mathcal{C}_{*} U_{k-1}\left(Y_{g}\right)\right) \rightarrow H_{k-1}\left(Q_{k-1}\right)$ are injections. Putting these facts together, we obtain that if $k \leq 2 g$, then $H_{k}\left(\mathcal{C}_{*} U_{k}\left(Y_{g}\right)\right)$ is isomorphic to the kernel of the composite map

$$
\partial_{k}: H_{k}\left(Q_{k}\right) \rightarrow H_{k-1}\left(\mathcal{C}_{*} U_{k-1}\left(Y_{g}\right)\right) \hookrightarrow H_{k-1}\left(Q_{k-1}\right) .
$$

Using Lemma 4.4 completes the proof of the lemma for these values of $i, g$ and $k$.

(I.3) The case $k \leq 2 g$ and $i<k$ : Because of (16) it is sufficient to compute $H_{k-1}\left(\mathcal{C}_{*} U_{k}\left(Y_{g}\right)\right)$. This can be read off from the following diagram with exact rows:

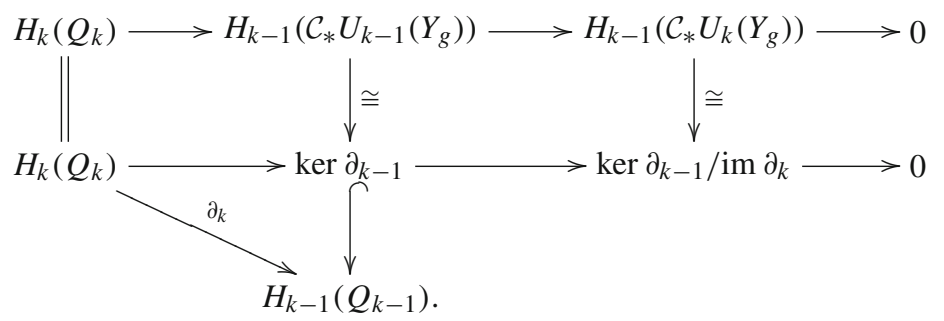

Now we turn to the cases when $k>2 g$. The lemma claims that under this assumption

$$
H_{i}\left(U_{k}\left(Y_{g}\right)\right)= \begin{cases}\mathbb{Z} & \text { if } i \text { is even and } i \leq 2 k-2 g, \\ 0 & \text { else. }\end{cases}
$$

Using Lemma 4.4 and the short exact sequence of chain complexes $0 \rightarrow \mathcal{C}_{*} U_{k-1}\left(Y_{g}\right) \rightarrow$ $\mathcal{C}_{*} U_{k}\left(Y_{g}\right) \rightarrow Q_{k} \rightarrow 0$ we see that, for $k>2 g$, we have isomorphisms

$$
H_{i}\left(\mathcal{C}_{*} U_{k-1}\left(Y_{g}\right)\right) \cong H_{i}\left(\mathcal{C}_{*} U_{k}\left(Y_{g}\right)\right), \quad i \neq 2 k-2 g-1,2 k-2 g .
$$

(II.1) The case $k>2 g$ and $i \geq 2 k-2 g+1$ : In this case, $i>2 g$. It follows from case (I.1) above that $H_{i}\left(\mathcal{C}_{*} U_{2 g}\left(Y_{g}\right)\right) \cong 0$, so we obtain from (18) that

$$
0 \cong H_{i}\left(\mathcal{C}_{*} U_{2 g}\left(Y_{g}\right)\right) \cong H_{i}\left(\mathcal{C}_{*} U_{2 g+1}\left(Y_{g}\right)\right) \cong \cdots \cong H_{i}\left(\mathcal{C}_{*} U_{k}\left(Y_{g}\right)\right),
$$

and consequently $H_{i}\left(\mathcal{C}_{*} U_{k}\left(Y_{g}\right)\right) \cong 0$.

(II.2) The case $k>2 g$ and $2 k-2 g-1 \leq i \leq 2 k-2 g$ : We know from case (II.1) that

$$
\begin{aligned}
H_{2 k-2 g}\left(\left(\mathcal{C}_{*} U_{k-1}\left(Y_{g}\right)\right)\right) & \cong 0, \\
H_{2 k-2 g-1}\left(\left(\mathcal{C}_{*} U_{k-1}\left(Y_{g}\right)\right)\right) & \cong 0 .
\end{aligned}
$$

From the short exact sequence $0 \rightarrow \mathcal{C}_{*} U_{k-1}\left(Y_{g}\right) \rightarrow \mathcal{C}_{*} U_{k}\left(Y_{g}\right) \rightarrow Q_{k} \rightarrow 0$ together with Lemma (4.4), we conclude

$$
\begin{aligned}
H_{2 k-2 g}\left(\left(\mathcal{C}_{*} U_{k}\left(Y_{g}\right)\right)\right) & \cong H_{2 k-2 g}\left(Q_{k}\right) \cong \mathbb{Z}, \\
H_{2 k-2 g-1}\left(\left(\mathcal{C}_{*} U_{k}\left(Y_{g}\right)\right)\right) & \cong 0 .
\end{aligned}
$$


(II.3) The case $k>2 g, i \leq 2 k-2 g-2$ : This follows from case II.2 together with the isomorphisms (18).

Before we prove our main theorem we need one more technical lemma. For $1 \leq m \leq g$, let

$$
\sigma_{m}:=\sum_{1 \leq i_{1}<i_{2}<\cdots<i_{m} \leq g} e_{i_{1}} f_{i_{1}} \ldots e_{i_{m}} f_{i_{m}}
$$

be the elementary symmetric polynomials in the $g$ products $e_{i} f_{i}$. Note that $\lambda=-\partial \sigma_{1}$ where $\lambda$ was defined before Eq. (14).

Lemma 4.6 The cohomology of the complex

$$
\mathbf{K}_{0} \stackrel{\lambda}{\rightarrow} \mathbf{K}_{1} \stackrel{\lambda}{\rightarrow} \cdots \mathbf{K}_{2 g-1} \stackrel{\lambda}{\rightarrow} \mathbf{K}_{2 g}
$$

is $\mathbb{Z}$ in odd degrees $2 m+1$, where $2 g-1 \geq 2 m+1 \geq 3$, and 0 elsewhere; $H^{2 m+1}\left(\mathbf{K}_{*}\right)$ is generated by $\lambda \sigma_{m}$.

Proof We consider the complex

$$
\mathcal{C}_{0}\left(X_{2 g}\right) \stackrel{\lambda}{\rightarrow} \mathcal{C}_{1}\left(X_{2 g}\right) \stackrel{\lambda}{\rightarrow} \cdots \mathcal{C}_{2 g-1}\left(X_{2 g}\right) \stackrel{\lambda}{\rightarrow} \mathcal{C}_{2 g}\left(X_{2 g}\right)
$$

Let $\mathbf{B}_{0}$ be the image of $\partial: \mathcal{C}_{1}\left(X_{2 g}\right) \rightarrow \mathcal{C}_{0}\left(X_{2 g}\right)$. There is a short exact sequence of cochain complexes:

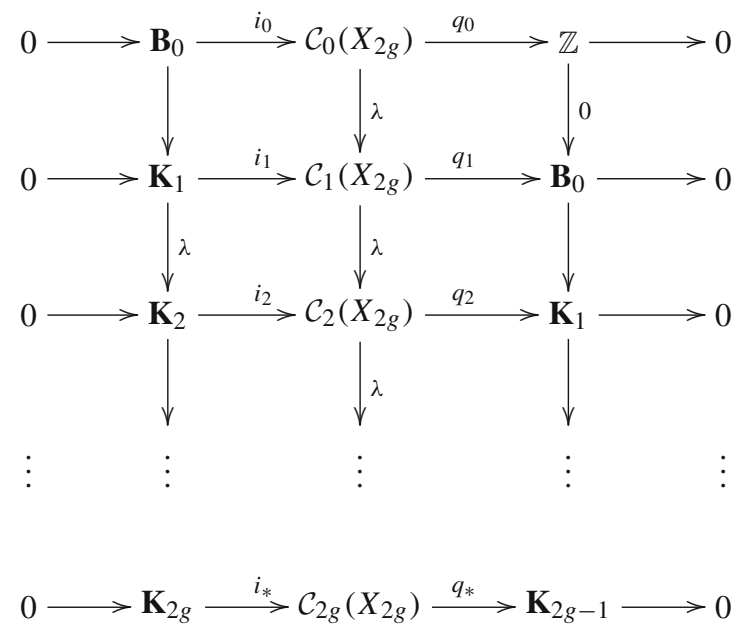

Let $\mathbf{H}^{i}$ be the cohomology of the cochain complex

$$
0 \rightarrow \mathbf{B}_{0} \rightarrow \mathbf{K}_{1} \stackrel{\lambda}{\rightarrow} \mathbf{K}_{2} \rightarrow \cdots \rightarrow \mathbf{K}_{2 g} \rightarrow 0
$$


The short exact sequence of complexes above induces a long exact sequence of cohomology groups:
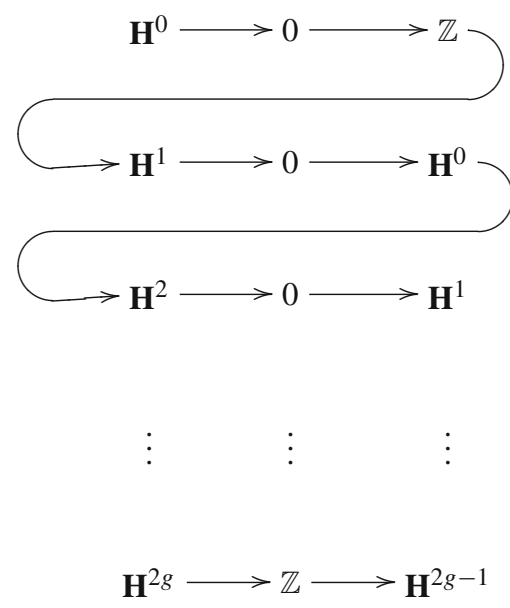

It follows inductively that

$$
\mathbf{H}^{i}= \begin{cases}\mathbb{Z} & \text { if } 1 \leq i \leq 2 g-1, \text { and } i \text { odd }, \\ 0 & \text { else. }\end{cases}
$$

Actually, we can find a generator for each of the homology groups inductively. The first claim is that $\mathbf{H}^{1}$ is generated by $\lambda$. This follows from the long exact sequence (20), by the definition of the boundary map (see e.g. [24] Chapter 2). Assume inductively that $\mathbf{H}^{2 m-1}$ is generated by $\lambda \sigma_{m-1}$. The group $\mathbf{H}^{2 m+1}$ is generated by the image of $\lambda \sigma_{m-1}$ under the boundary map of the long exact sequence of (20). To compute this boundary, we first find a chain in $\mathcal{C}_{2 g-1}\left(X_{2 g}\right)$ that maps to the cycle $\lambda \sigma_{m-1} ; \sigma_{m}$ is up to sign such a chain:

$$
\begin{aligned}
\partial \sigma_{m} & =\partial \sum_{1 \leq i_{1}<i_{2}<\cdots<i_{m} \leq g} e_{i_{1}} f_{i_{1}} \ldots e_{i_{m}} f_{i_{m}} \\
& =-\lambda \sum_{1 \leq i_{1}<i_{2}<\cdots<i_{m-1} \leq g} e_{i_{1}} f_{i_{1}} \ldots e_{i_{m-1}} f_{i_{m-1}} \\
& =-\lambda \sigma_{m-1} .
\end{aligned}
$$

By the definition of the boundary map, $\mathbf{H}^{2 m+1}$ is generated by the product of $\lambda$ with $\sigma_{m}$, which completes the induction.

This finishes the computation of the cohomology of the cochain complex (19). This cohomology agrees with the cohomology of $\mathbf{K}_{*}$ except in degrees 0 and 1 . To complete the proof of the lemma, we now only have to check that it is true for those two dimensions as well. But this also follows from our computation, and the fact that since $\mathbf{K}_{0}=\mathcal{C}_{0}\left(X_{2 g}\right)$, there is a short exact sequence

$$
0 \rightarrow \mathbf{B}_{0} \rightarrow \mathbf{K}_{0} \rightarrow \mathbb{Z} \rightarrow 0
$$

At this point we are able to prove the main theorem of this section. 
Theorem 4.7 Let $k \geq 2$. Then

$$
H_{i}\left(\widetilde{\operatorname{Sym}^{k}(\Sigma)}\right) \cong H_{i}\left(U_{k}(Y)\right) \cong \begin{cases}\mathbb{Z} & \text { i even, } i \neq k \text { and } i \leq \max (2 k-2 g, k), \\ \mathbf{K}_{k} / \lambda \mathbf{K}_{k-1} \oplus \mathbb{Z} & i=k \text { even }, k \leq 2 g, \\ \lambda \mathbf{K}_{k} & i=k \text { odd }, k \leq 2 g, \\ 0 & \text { else. }\end{cases}
$$

Proof In the notations of Lemma 4.5 we have to compute the groups ker $\partial_{k}$ and ker $\partial_{k} / \mathrm{im} \partial_{k-1}$.

Consider the following commutative diagram (notation as in the proof of Lemma 4.6):

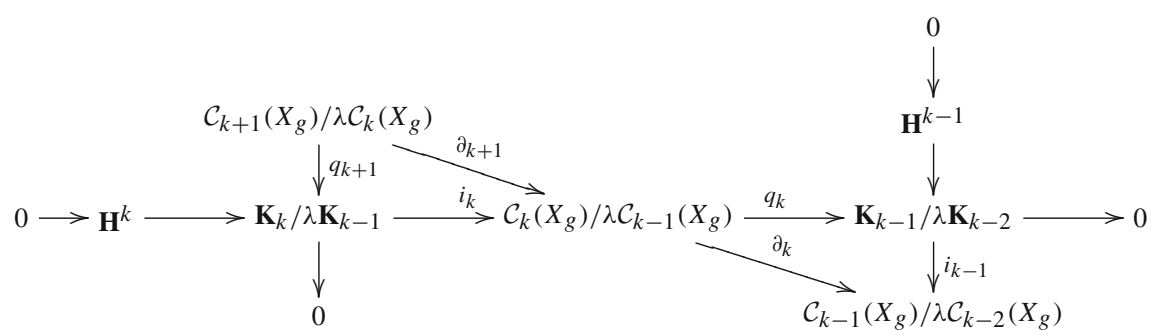

We claim that, if $k \geq 2$, then the two columns and the horizontal row are exact. The exactness of the left column follows since $q_{k+1}: \mathcal{C}_{k+1} \rightarrow \mathbf{K}_{k}$ is surjective. The exactness of the horizontal row and the right vertical column is easy to check by diagram chasing. For instance, to prove exactness of the horizontal row at $\mathbf{K}_{k} / \lambda \mathbf{K}_{k-1}$, if $a \in \mathbf{K}_{k}$ such that $[a] \in \mathbf{K}_{k} / \lambda \mathbf{K}_{k-1}$ is in the kernel of $i_{k}$, then there is a $b \in \mathcal{C}_{k-1}\left(X_{g}\right)$ such that $a=\lambda b \in \mathbf{K}_{k}$. Since $\lambda a=\lambda^{2} b=0, a$ represents a homology class in $\mathbf{H}^{k}$.

From the diagram above follows that

$$
\begin{aligned}
H_{k}\left(U_{k}\left(Y_{g}\right)\right) & =\operatorname{ker} \partial_{k}=q_{k}^{-1}\left(\mathbf{H}^{k-1}\right) \cong \operatorname{im}\left(i_{k}\right) \oplus \mathbf{H}^{k-1} \\
& = \begin{cases}\mathbb{Z} \oplus \mathbf{K}_{k} / \lambda \mathbf{K}_{k-1} & \text { if } k \text { is even, } \\
\left(\mathbf{K}_{k} / \lambda \mathbf{K}_{k-1}\right) / \mathbf{H}^{k} \cong \lambda \mathbf{K}_{k} & \text { if } k \text { is odd } .\end{cases}
\end{aligned}
$$

This proves the theorem for the case $k=i$.

In the case $k \neq i$, the same computation proves that, if $i<k \leq 2 g$, one has

$$
H_{i}\left(U_{k}\left(Y_{g}\right)\right)=\operatorname{ker} \partial_{k} / \operatorname{im}\left(i_{k}\right) \cong \mathbf{H}^{i-1} \cong \begin{cases}\mathbb{Z} & \text { if } k \text { is even }, \\ 0 & \text { if } k \text { is odd }\end{cases}
$$

Together with Lemma 4.5, this completes the proof of the theorem.

We now wish to make a few remarks on these groups:

Lemma 4.8 (1) $\mathbf{K}_{k} / \lambda \mathbf{K}_{k}$ and $\lambda \mathbf{K}_{k}$ are free Abelian groups.

(2) $\mathbf{K}_{2 g} / \lambda \mathbf{K}_{2 g-1}=0$ and $\lambda \mathbf{K}_{2 g-1}=0$.

(3) If $2 \leq k \leq 2 g-2$, then neither $\lambda \mathbf{K}_{k}$ nor $\mathbf{K}_{k} / \lambda \mathbf{K}_{k-1}$ are finitely generated.

Proof (1) $\lambda \mathbf{K}_{k} \subset \mathbf{K}_{k+1} \subset \mathcal{C}_{k+1}\left(X_{2 g}\right)$, so as a subgroup of a free Abelian group, it is free Abelian. The canonical short exact sequence

$$
0 \rightarrow \mathbf{H}_{k} \rightarrow \mathbf{K}_{k} / \lambda \mathbf{K}_{k-1} \rightarrow \lambda \mathbf{K}_{k} \rightarrow 0
$$

splits, since $\lambda \mathbf{K}_{k}$ is a free Abelian group. It follows that $\mathbf{K}_{k} / \lambda \mathbf{K}_{k}$ is also a free Abelian group. 
(2) Since $\mathbf{K}_{2 g}=0$, we obviously have that $\mathbf{K}_{2 g} / \lambda \mathbf{K}_{2 g-1}$ is trivial. Let $a \in \mathbf{K}_{2 g-1}$; we want to show that $\lambda a$ is trivial. But $a=\partial b$ for some $b \in \mathcal{C}_{2 g}$, so $\lambda a=\lambda \partial b=\partial(\lambda b)$. Since $\lambda b \in \mathcal{C}_{2 g+1}=0$, this class is zero.

(3) We need to show that if $2 \leq k \leq 2 g-2$, then $\lambda \mathbf{K}_{k}$ is a nontrivial group. Pick integers $1<i_{1}<i_{2}<\cdots<i_{m} \leq g, 1<j_{1}<j_{2}<\cdots<j_{n} \leq g$ such that $m+n=k$. This can be done, since $k \leq 2 g-2$. Let $a=e_{1} e_{i_{1}} \ldots e_{i_{m}} f_{i_{1}} \ldots f_{j_{n}} \in \mathcal{C}_{k+1}$. The point is that this is a monomial in $e$ and $f$ such that $e_{1}$ occurs, but $f_{1}$ does not occur. We claim that $\lambda \partial a$ is a nontrivial element of $\lambda \mathbf{K}_{k}$. To see that it is nontrivial, consider the homomorphism $F: \mathcal{C}_{*} \rightarrow \Lambda\left[e_{1}, \ldots, e_{g}, f_{1}, \ldots, f_{g}\right]$ given by $F\left(x_{i}\right)=1, i \neq 1, F\left(x_{1}\right)=0, F\left(y_{i}\right)=1$, $F\left(e_{i}\right)=e_{i}$ and $F\left(f_{i}\right)=f_{i}$. We compute

$$
F(\lambda \partial a)=F(\lambda) F(\partial a)=-f_{1} \cdot e_{i_{1}} \ldots e_{i_{m}} f_{i_{1}} \ldots f_{j_{n}} \neq 0 .
$$

It follows that $\lambda \mathbf{K}_{k} \neq 0$. Moreover, $\lambda \mathbf{K}_{k} \subset \mathcal{C}_{n+1}$ as a submodule over the group ring $\mathbb{Z}[\pi]$. There are no zero divisors in this ring, so any nontrivial element of $\mathcal{C}_{n+1}$ generates a copy of $\mathbb{Z}[\pi]$, which is not finitely generated as an Abelian group. It follows that $\lambda \mathbf{K}_{k}$ cannot be finitely generated.

Remark 4.9 For even $k$, we have a short exact sequence of modules

$$
0 \rightarrow \mathbf{K}_{k} / \lambda \mathbf{K}_{k-1} \rightarrow H_{k}\left(U_{k}\left(X_{2 g}\right)\right) \rightarrow \mathbb{Z} \rightarrow 0 .
$$

This sequence is split as a short exact sequence of groups, but it is not split as a sequence of $\mathbb{Z}\left[x_{1}^{ \pm 1}, \ldots, x_{g}^{ \pm 1}, y_{1}^{ \pm 1}, \ldots, y_{g}^{ \pm 1}\right]$-modules. In particular, for $k=2$, Lemma 9 of [30] shows that for $g=2$ we have $H_{2}\left(U_{2}\left(X_{2 g}\right)\right) \cong \mathbb{Z}\left[x_{1}^{ \pm 1}, x_{2}^{ \pm 1}, y_{1}^{ \pm 1}, y_{2}^{ \pm 1}\right]$, which does not contain a submodule isomorphic to $\mathbb{Z}$. However, the result is compatible with ours. If

$$
\eta: \mathbb{Z}\left[x_{1}^{ \pm 1}, x_{2}^{ \pm 1}, y_{1}^{ \pm 1}, y_{2}^{ \pm 1}\right] \rightarrow \mathbb{Z}
$$

is the augmentation map, there is an isomorphism $\alpha: \mathbf{K}_{k} / \lambda \mathbf{K}_{k-1} \rightarrow \operatorname{ker}(\eta)$.

For the purposes of this paper, the main outcome of our homology computations is the following consequence of Lemma 4.8:

Corollary 4.10 Let $\Sigma$ be a closed, oriented surface of genus $g>1$. Assume that $2 \leq k \leq$ $2 g-2$. Let $\widehat{\operatorname{Sym}^{k}(\Sigma)}$ denote the universal cover of the $k$-fold symmetric product of $\Sigma$. The homology group $H_{k}\left(\widehat{\operatorname{Sym}^{k}(\Sigma)} ; \mathbb{Z}\right)$ is a free Abelian group, but it is not finitely generated.

\section{The homotopy type of $\widetilde{\operatorname{Sym}^{k}(\Sigma)}$}

In this section, we determine the homotopy type of the universal cover of the symmetric products of a closed oriented surface. Although not directly relevant to the proof of our main result, we include this calculation for the sake of completeness.

\section{Theorem 5.1}

$$
\pi_{i}\left(U_{k}\left(X_{n}\right)\right)= \begin{cases}0 & \text { if } i<k \text { or if } n \leq k, \\ \mathbf{K}_{k} & \text { if } i=k<n .\end{cases}
$$

Furthermore, $U_{k}\left(X_{n}\right)$ is homotopy equivalent to a wedge of spheres of dimension $k$. 
Proof According to Lemma 4.3, $U_{k}\left(X_{n}\right)$ has trivial homology in dimensions less than $k$. Since it is also simply connected by definition, Hurewicz's theorem says that the Hurewicz map

$$
h: \pi_{k}\left(U_{k}\left(X_{n}\right)\right) \rightarrow H_{k}\left(U_{k}\left(X_{n}\right)\right)
$$

is an isomorphism. The statements about homotopy groups follow from this and the computation in Lemma 4.3 of the cellullar homology of $U_{k}(X)$. To prove the last statement, chose a family of generators $\left\{f_{\alpha}\right\}_{\alpha \in A}$ for $\pi_{k}\left(U_{k}(X)\right)$. This family defines a homology equivalence $f=\vee f_{\alpha}: \vee S_{\alpha}^{k} \rightarrow U_{k}(X)$. Since both its source and the target are simply connected, $f$ is a homotopy equivalence.

Remark 5.2 Theorem 5.1 is compatible with [31], Lemma 3, which states that $\overline{\operatorname{Sym}^{k}(X)}$ is homeomorphic to the $k$-skeleton of the torus $\mathbb{T}^{2 g}$.

Lemma 5.3 Let $m:=\max \{k-g,[k / 2]\}$. There is a map $f: \vee_{i \in I} S^{k} \rightarrow U_{k}\left(Y_{g}\right)$ such that the mapping cone $C(f)$ of $f$ is homotopy equivalent to $\mathbb{C P}^{m}$. If $2 \leq k \leq 2 g-2$, the index set I is infinite.

Proof If $k \geq 2 g$, we know from a theorem by Mattuck [40] that $U_{k}\left(Y_{g}\right) \simeq \widetilde{\operatorname{Sym}^{k}\left(\Sigma_{g}\right)}$ is homotopy equivalent to $\mathbb{C P}^{k-g}$. We only have to consider the case that $k<2 g$. The stabilisation map defines a map

$$
s: U_{k}\left(Y_{g}\right) \rightarrow U_{2 g}\left(Y_{g}\right) \simeq \mathbb{C P}^{g} \subset \mathbb{C P}^{\infty} .
$$

This map is an isomorphism on homology in dimensions $\leq k-1$, and by Lemma 4.3 and Theorem 4.7 we have an exact sequence

$$
H_{k}\left(U_{k}\left(X_{2 g}\right) \stackrel{i_{*}}{\rightarrow} H_{k}\left(U_{k}\left(Y_{g}\right)\right) \rightarrow H_{k}\left(\mathbb{C P}^{\infty}\right) \rightarrow 0 .\right.
$$

We can chose a free subgroup of $H_{k}\left(U_{k}\left(X_{2 g}\right)\right)$ that maps isomorphically to the image of $H_{k}\left(U_{k}\left(X_{2 g}\right)\right)$ inside $H_{k}\left(U_{k}\left(Y_{g}\right)\right)$. The Hurewicz map identifies this subgroup with a free subgroup of $\pi_{k}\left(U_{k}\left(X_{2 g}\right)\right)$. Let $I$ be a set of generators for this subgroup. Each generator corresponds to a homotopy class of based maps $S^{k} \rightarrow U_{k}\left(X_{2 g}\right)$. Together, they define a map $f: \vee_{i \in I} S^{k} \rightarrow U_{k}\left(Y_{g}\right)$ such that $s \circ f$ is homotopically trivial. We intend to show that $C(f)$ is homotopy equivalent to $\mathbb{C P}^{m}$. The long exact homology sequence of the cofibration defining $C(f)$ already shows that at least its homology is correct:

$$
H_{i}(C(f)) \cong \begin{cases}\mathbb{Z} & \text { if } 0 \leq i \leq 2 m \text { and } i \text { is even, } \\ 0 & \text { else. }\end{cases}
$$

The homotopy trivialisation of the composite $s \circ f$ defines a map $s^{\prime}$ from the cofibre $C(f)$ to $\mathbb{C P}^{\infty}$. By obstruction theory, the obstructions to factoring this map over $\mathbb{C P}^{m}$ lie in the groups $H^{j}\left(C(f), \pi_{j-1}\left(S^{2 m+1}\right)\right)$. Since these groups are all trivial, we can find a map $h: C(f) \rightarrow \mathbb{C P}^{m}$ such that $s^{\prime}$ is homotopic to $i \circ h$. It follows that $h$ induces isomorphism on all homology groups. The assertion in the theorem now follows from this and from Whitehead's theorem.

This result is sufficient to determine the homotopy type of $U_{k}\left(Y_{g}\right)$.

Theorem 5.4 There are homotopy equivalences

$$
\widetilde{\operatorname{Sym}^{k}\left(\Sigma_{g}\right)} \simeq U_{k}\left(Y_{g}\right) \simeq \mathbb{C P}^{m} \vee\left(\vee_{i \in I} S^{k}\right),
$$

where $m$ and $I$ are as in Lemma 5.3. 
Proof According to Lemma 5.3 we have a cofibration sequence up to homotopy

$$
\vee_{i \in I} S^{k} \stackrel{f}{\rightarrow} U_{k}\left(Y_{g}\right) \stackrel{h}{\rightarrow} \mathbb{C P}^{m} .
$$

We construct a splitting $s$, up to homotopy, of the map $h: U_{k}(Y) \rightarrow \mathbb{C P}^{m}$. That is, a map $s: \mathbb{C P}^{m} \rightarrow U_{k}\left(Y_{g}\right)$ such that the composite $s \circ f$ is homotopic to the identity. The existence of this splitting is guaranteed by obstruction theory, since the obstructions to the splitting are in the trivial groups $H^{i}\left(\mathbb{C P}^{m}, \pi_{i}\left(C(f), U_{n}\left(\Sigma_{g}\right)\right)\right.$.

Consider the diagram, commutative up to homotopy

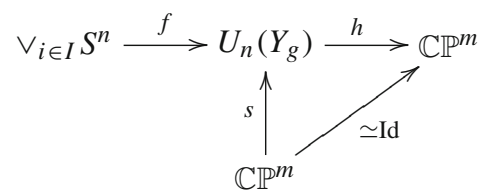

The top row is a cofibration up to homotopy. It follows that the map $f \vee s: \vee_{i \in I} S^{n} \vee \mathbb{C P}^{m} \rightarrow$ $U_{n}\left(Y_{g}\right)$ is a homotopy equivalence.

\section{Rigidity and holomorphic bisectional curvature}

After the topological digression of the three preceding sections, we are equipped to give a proof of Theorem 1.1. We start by recalling the following rigidity result of Mok [41,42]:

Theorem 6.1 Suppose that $\tilde{M}$ is the universal cover of a compact Kähler manifold $M$ with nonnegative holomorphic bisectional curvature. Then, for suitable $m, m_{1}, \ldots, m_{r}, s \in \mathbb{N}_{0}$, there is a biholomorphic isometry of $\tilde{M}$, equipped with the Kähler metric pulled back from $M$, to the product of Kähler manifolds

$$
\left(\mathbb{C}^{m}, e\right) \times \prod_{i=1}^{r}\left(\mathbb{C P}^{m_{i}}, \vartheta_{i}\right) \times \prod_{j=1}^{s}\left(H_{j}, h_{j}\right),
$$

where e is the Euclidean metric, $\vartheta_{i}$ are Kähler metrics of nonnegative holomorphic bisectional curvature, and $h_{j}$ are canonical metrics on compact irreducible Hermitian symmetric spaces $H_{j}$ of rank $>1$.

Recall that a Hermitian symmetric space is a Riemannian symmetric space $G / H$ for a Lie group $G$, equipped with a parallel complex structure with respect to which the metric is Hermitian; so the complex structure is preserved by $H$ and there is an underlying Kähler structure.

The following is an easy consequence of the classification:

Proposition 6.2 Let $M$ be a compact Kähler manifold with nonnegative holomorphic bisectional curvature. Then the total singular homology $H_{*}(\tilde{M} ; \mathbb{Z})$ of its universal cover is finitely generated (as an Abelian group).

Proof According to the decomposition of Theorem 6.1, the universal cover $\tilde{M}$ is homotopy equivalent to a product

$$
\prod_{i=1}^{r} \mathbb{C P}^{m_{i}} \times \prod_{j=1}^{s} H_{j}
$$


All factors are compact manifolds, so their product is also a compact manifold. But the homology of a compact manifold is finitely generated in every dimension. (see e.g. [24], Appendix, Corollary A9 and Corollary A8).

Proof of Theorem 1.1 We fix positive integers $g$ and $k$ such that $1<k<2 g-1$, and consider the case of the moduli space of vortices $M=\operatorname{Sym}^{k}\left(\Sigma_{g}\right)$. Combining Proposition 6.2 with Corollary 4.10, Theorem 1.1 follows immediately in the situation where $k>1$. But as we saw in the end of the Introduction, the case $k=1$ is trivial.

\section{Vortices on hyperelliptic surfaces}

In this final section, we let $\Sigma$ be a hyperelliptic Riemann surface of genus $g \geq 2$ - for example, any smooth complex curve of genus $g=2$. Recall that $\operatorname{Sym}^{k}(\Sigma)$ is a manifold such that, for $k \geq 2, \pi_{1}\left(\operatorname{Sym}^{k}(\Sigma)\right) \cong \mathbb{Z}^{2 g}$ (see Lemma 2.2). In [31], the second homotopy group of this space is discussed. In particular, Lemma 9 of this paper says that

$$
\pi_{2}\left(\operatorname{Sym}^{2}(\Sigma)\right)=\mathbb{Z}\left[t_{i}, t_{i}^{-1}\right], \quad 1 \leq i \leq 4 .
$$

In the present work, we have generalised this formula to higher homotopy groups in Theorem 5.1.

The hyperelliptic condition can be characterised by saying that $\Sigma$ admits a holomorphic involution

$$
\sigma: \Sigma \longrightarrow \Sigma
$$

such that $p: \Sigma \rightarrow \Sigma /\{p \sim \sigma(p)\}$ is a branched cover over $S^{2} \cong \Sigma /\{p \sim \sigma(p)\}$; whenever such map $\sigma$ exists, it is unique. Given $\sigma$ or $p$, there is an obvious holomorphic map

$$
\alpha: S^{2} \longrightarrow \operatorname{Sym}^{2}(\Sigma)
$$

which sends a point of the base $S^{2}$ of $p$ to the two (possibly equal) points on $\Sigma$ covering it. The domain of this map can be interpreted as a projective line parametrising all effective divisors in a $g_{2}^{1}$ (i.e. pencil of degree two, cf. [1]) associated to the hyperelliptic structure. In Lemma 5 of Ref. [30], the image $h_{*}([\alpha])$ in $H_{2}\left(\operatorname{Sym}^{2}(\Sigma) ; \mathbb{Z}\right)$ of the homotopy class represented by (24) under the Hurewicz map is computed. It is further found that this image is a non-torsion element of the second homology group $H_{2}\left(\operatorname{Sym}^{2}(\Sigma) ; \mathbb{Z}\right)$, so $[\alpha]$ and all its integral multiples define nontrivial elements of the second homotopy group $\pi_{2}\left(\operatorname{Sym}^{2}(\Sigma)\right)$.

Notice that the hyperelliptic involution $\sigma$ induces another involution map

$$
\sigma^{(2)}: \operatorname{Sym}^{2}(\Sigma) \longrightarrow \operatorname{Sym}^{2}(\Sigma),
$$

defined on degree two divisors on $\Sigma$ by $x+y \mapsto \sigma(x)+\sigma(y)$. Clearly, the set of fixed points of this map $\sigma^{(2)}$ is the image of the map $\alpha$ in (24). We have the following result:

Proposition 7.1 The image of the map $\alpha$ is a noncontractible holomorphic 2-sphere in the moduli space of two vortices on $\Sigma$. If the map $\sigma^{(2)}$ is an isometry of the vortex metric on $\operatorname{Sym}^{2}(\Sigma)$, then this 2-sphere is totally geodesic.

Proof The argument we have just given above already impies that $\alpha\left(S^{2}\right)$ is not contractible in $\operatorname{Sym}^{2}(\Sigma)$. Since $\alpha$ is holomorphic, it embeds the 2 -sphere as a complex submanifold. It is a general result in Riemannian geometry that, if the the set of fixed points of an isometry form a submanifold, it is necessarily totally geodesic — this follows easily from the Picard-Lindelöf theorem applied to geodesic flow (see Appendix A in [44]). 
The induced $L^{2}$-metric gives a shape to this 2-sphere, whose geometry would be interesting to understand. In [37], it is shown that, as $\tau \rightarrow \frac{4 \pi k}{\operatorname{Vol}\left(\Sigma_{g}\right)}$ (the "dissolving limit"), the metric degenerates: null-vectors appear precisely along the tangent directions to this 2-sphere as the limit is attained. A number of natural questions can be asked, for example: which metrics on $\Sigma$ will ensure that this sphere is round, or that it admits nontrivial isometries?

Acknowledgments NMR would like to thank João Baptista for discussions in the early stages of this project, Indranil Biswas for clarifying some questions, and Martin Speight for a helpful comment. This work was partially supported by CTQM, University of Aarhus, and by the European Commission in the framework of the Marie Curie project MTKD-CT-2006-042360, hosted by the Jagiellonian University in Cracow.

Open Access This article is distributed under the terms of the Creative Commons Attribution License which permits any use, distribution, and reproduction in any medium, provided the original author(s) and the source are credited.

\section{References}

1. Arbarello, E., Cornalba, M., Griffiths, P.A., Harris, J.: Geometry of Algebraic Curves, vol. 1. Springer, Berlin (1985)

2. Baptista, J.M.: On the $L^{2}$-metric of vortex moduli spaces. Nucl. Phys. B 844, 308-333 (2011)

3. Bertram, A., Daskalopoulos, G., Wentworth, R.: Gromov invariants for holomorphic maps from Riemann surfaces to Grassmannians. J. Am. Math. Soc. 9, 529-571 (1996)

4. Bertram, A., Thaddeus, M.: On the quantum cohomology of a symmetric product of an algebraic curve. Duke Math. J. 108, 329-362 (2001)

5. Biswas, I.: On Kähler structures over symmetric products of a Riemann surface. Proc. Am. Math. Soc. 141, 1487-1492 (2013)

6. Biswas, I.: On the curvature of symmetric products of a compact Riemann surface. Arch. Math. (Basel) 100, 413-415 (2013)

7. Biswas, I., Schumacher, G.: Determinant bundle, Quillen metric, and Petersson-Weil form on moduli spaces. Geom. Funct. Anal. 9, 226-255 (1999)

8. Biswas, I., Schumacher, G.: Geometry of moduli spaces of Higgs bundles. Commun. Anal. Geom. 14, 765-793 (2006)

9. Biswas, I., Schumacher, G.: Kähler structure on moduli spaces of principal bundles. Differ. Geom. Appl. 25, 136-146 (2007)

10. Biswas, I., Schumacher, G.: Coupled vortex equations and moduli: deformation theoretic approach and Kähler geometry. Math. Ann. 343, 825-851 (2009)

11. Bradlow, S.B.: Vortices in holomorphic line bundles over closed Kähler manifolds. Commun. Math. Phys. 135, 1-17 (1990)

12. Bradlow, S.B., García-Prada, O.: Stable triples, equivariant bundles and dimensional reduction. Math. Ann. 304, 225-252 (1996)

13. Cieliebak, K., Gaio, R.A., Mundet i Riera, I., Salamon, D.A.: The symplectic vortex equations and invariants of Hamiltonian group actions. J. Sympl. Geom. 1, 543-645 (2001)

14. Collie, B., Tong, D.: The dynamics of Chern-Simons vortices. Phys. Rev. D 78, 065013 (2008)

15. Donaldson, S.K.: Topological field theories and formulae of Casson and Meng-Taubes. Geom. Topol. Monogr. 2, 87-102 (1999)

16. Eastwood, M.G., Romão, N.M.: A combinatorial formula for homogeneous moments. Math. Proc. Camb. Philos. Soc. 142, 153-160 (2007)

17. Fang, F.: Kähler manifolds with almost non-negative bisectional curvature. Asian J. Math. 6, 385-398 (2002)

18. Fulton, W.: Algebraic Topology: A First Course. Springer, Berlin (1997)

19. García-Prada, O.: A direct existence proof for the vortex equations over a compact Riemann surface. Bull. Lond. Math. Soc. 26, 88-96 (1992)

20. García-Prada, O.: Dimensional reduction of stable bundles, vortices and stable pairs. Int. J. Math. 5, 1-52 (1994)

21. Gel'fand, S.I., Manin, Yu.: Methods of Homological Algebra. Springer, Berlin (2003)

22. Goldberg, S.I., Kobayashi, S.: Holomorphic bisectional curvature. J. Differ. Geom. 1, 225-233 (1967)

23. Griffiths, P., Harris, J.: Principles of Algebraic Geometry. Wiley, New York (1978) 
24. Hatcher, A.: Algebraic Topology. Cambridge University Press, Cambridge (2002)

25. Hitchin, N.J.: The self-duality equations on a Riemann surface. Proc. Lond. Math. Soc. 55, 59-126 (1987)

26. Hitchin, N.J.: $L^{2}$-cohomology of hyperkähler quotients. Commun. Math. Phys. 211, 153-165 (2000)

27. Itoh, M.: Geometry of anti-self-dual connections and Kuranishi map. J. Math. Soc. Jpn. 40, 9-33 (1988)

28. Jaffe, A., Taubes, C.: Vortices and Monopoles. Birkhäuser, Boston (1980)

29. Jost, J., Peng, X.-W.: The geometry of moduli spaces of stable vector bundles over Riemann surfaces. In: Ferus, D., Simon, U., Pinkall, U. (eds.) Global Differential Geometry and Global Analysis (Berlin, 1990), Lecture Notes in Mathematics, vol. 1481, Springer, Berlin, pp. 79-96 (1991)

30. Kallel, S.: Some remarks on symmetric products of curves. arXiv:math/0402267v2

31. Kallel, S., Salvatore, P.: Symmetric products of two dimensional complexes. In: Ádem, A., González, J., Pastor, G. (eds.) Recent Developments in Algebraic Topology (San Miguel de Allende 2003), Contemporary Mathematics, vol. 407, pp. 147-161 (2006)

32. Lück, W.: $L^{2}$-invariants: Theory and Applications to Geometry and K-Theory. Springer, Berlin (2002)

33. Macdonald, I.G.: Symmetric products of an algebraic curve. Topology 1, 319-343 (1962)

34. MacLane, S.: Homology. Springer, Berlin (1975)

35. Manton, N.S.: First-order vortex dynamics. Ann. Phys. 256, 114-131 (1997)

36. Manton, N.S., Nasir, S.M.: Volume of vortex moduli spaces. Commun. Math. Phys. 199, 591-604 (1999)

37. Manton, N.S., Romão, N.M.: Vortices and Jacobian varieties. J. Geom. Phys. 61, 1135-1155 (2011)

38. Manton, N.S., Speight, J.M.: Asymptotic interactions of critically coupled vortices. Commun. Math. Phys. 236, 535-555 (2003)

39. Manton, N., Sutcliffe, P.: Topological Solitons. Cambridge University Press, Cambridge (2004)

40. Mattuck, A.: On symmetric products of curves. Proc. Am. Math. Soc. 13, 82-87 (1962)

41. Mok, N.: The uniformization theorem for compact Kähler manifolds of nonnegative holomorphic curvature. J. Differ. Geom. 27, 179-214 (1988)

42. Mok, N.: Metric Rigidity Theorems on Hermitian Locally Symmetric Manifolds. World Scientific, Singapore (1989)

43. Moss, I., Shiiki, N.: Quantum mechanics on moduli spaces. Nucl. Phys. B 565, 345-362 (2000)

44. Romão, N.M.: Dynamics of $\mathbb{C P}^{1}$ lumps on a cylinder. J. Geom. Phys. 54, 42-76 (2005)

45. Romão, N.M.: Gauged vortices in a background. J. Phys. A: Math. Gen. 38, 9127-9144 (2005)

46. Samols, T.M.: Vortex scattering. Commun. Math. Phys. 145, 149-180 (1992)

47. Segal, G.: Topology of the space of SU(2)-monopoles in $\mathbb{R}^{3}$. In: Andersen, J.E., Dupont, J., Pedersen, H., Swann, A. (eds.) Geometry and Physics (Aarhus 1995), Lecture Notes in Pure and Applied Mathematics, vol. 184, pp. 141-147. Marcel Dekker, New York (1997)

48. Segal, G., Selby, A.: The cohomology of the space of magnetic monopoles. Commun. Math. Phys. 177, 775-787 (1996)

49. Sen, A.: Dyon-monopole bound states, self-dual harmonic forms on the multi-monopole moduli space, and $\operatorname{SL}(2, \mathbb{Z})$ invariance in string theory. Phys. Lett. B 329, 217-221 (1994)

50. Sethi, S., Stern, M., Zaslow, E.: Monopole and dyon bound states in $N=2$ supersymmetric Yang-Mills theories. Nucl. Phys. B 329, 217-221 (1994)

51. Strachan, I.A.B.: Low-velocity scattering of vortices in a modified Abelian Higgs model. J. Math. Phys. 33, 102-110 (1992)

52. Stuart, D.M.A.: Dynamics of abelian Higgs vortices in the near Bogomolny regime. Commun. Math. Phys. 159, 51-91 (1994)

53. Takhtajan, L.A., Zograf, P.G.: On the geometry of moduli spaces of vector bundles over a Riemann surface. Math. USSR-Izv. 35, 83-100 (1990) 